\title{
Aligned-spin neutron-star-black-hole waveform model based on the effective-one-body approach and numerical-relativity simulations
}

\author{
Andrew Matas $\odot,{ }^{1}$ Tim Dietrich, ${ }^{2,3}$ Alessandra Buonanno, ${ }^{1,4}$ Tanja Hinderer, ${ }^{5,6}$ Michael Pürrer $\odot,{ }^{1}$ Francois Foucart, ${ }^{7}$ \\ Michael Boyle, ${ }^{8}$ Matthew D. Duez, ${ }^{9}$ Lawrence E. Kidder, ${ }^{8}$ Harald P. Pfeiffer, ${ }^{1}$ and Mark A. Scheel ${ }^{10}$ \\ ${ }^{1}$ Max Planck Institute for Gravitational Physics (Albert Einstein Institute), D-14476 Potsdam, Germany \\ ${ }^{2}$ Nikhef, Science Park 105, 1098 XG Amsterdam, Netherlands \\ ${ }^{3}$ Institute for Physics and Astronomy, University of Potsdam, \\ Karl-Liebknecht-Strasse 24/25, 14776 Potsdam, Germany \\ ${ }^{4}$ Department of Physics, University of Maryland, College Park, Maryland 20742, USA \\ ${ }^{5}$ GRAPPA, Anton Pannekoek Institute for Astronomy and Institute of High-Energy Physics, \\ University of Amsterdam, Science Park 904, 1098 XH Amsterdam, Netherlands \\ ${ }^{6}$ Delta Institute for Theoretical Physics, Science Park 904, 1090 GL Amsterdam, Netherlands \\ ${ }^{7}$ Department of Physics and Astronomy, University of New Hampshire, \\ 9 Library Way, Durham, New Hampshire 03824, USA \\ ${ }^{8}$ Cornell Center for Astrophysics and Planetary Science, Cornell University, Ithaca, New York 14853, USA \\ ${ }^{9}$ Department of Physics and Astronomy, Washington State University, Pullman, Washington 99164, USA \\ ${ }^{10}$ TAPIR, Walter Burke Institute for Theoretical Physics, MC 350-17, California Institute of Technology, \\ Pasadena, California 91125, USA
}

(Received 26 April 2020; accepted 6 August 2020; published 27 August 2020)

\begin{abstract}
After the discovery of gravitational waves from binary black holes (BBHs) and binary neutron stars (BNSs) with the LIGO and Virgo detectors, neutron-star black holes (NSBHs) are the natural next class of binary systems to be observed. In this work, we develop a waveform model for aligned-spin NSBHs combining a BBH baseline waveform (available in the effective-one-body approach) with a phenomenological description of tidal effects (extracted from numerical-relativity simulations) and correcting the amplitude during the late inspiral, merger and ringdown to account for the NS tidal disruption. In particular, we calibrate the amplitude corrections using NSBH waveforms obtained with the numerical-relativity spectral Einstein code (SpEC) and the SACRA code. The model was calibrated using simulations with NS masses in the range 1.2-1.4 $M_{\odot}$, tidal deformabilities up to 4200 (for a $1.2 M_{\odot} \mathrm{NS}$ ), and dimensionless BH spin magnitude up to 0.9. Based on the simulations used and on checking that sensible waveforms are produced, we recommend our model to be employed with a NS mass in the range 1-3 $M_{\odot}$, tidal deformability 0-5000, and (dimensionless) $\mathrm{BH}$ spin magnitude up to 0.9 . We also validate our model against two new, highly accurate NSBH waveforms with BH spin 0.9 and mass ratios 3 and 4, characterized by tidal disruption, produced with $\mathrm{SpEC}$, and find very good agreement. Furthermore, we compute the unfaithfulness between waveforms from NSBH, BBH, and BNS systems, finding that it will be challenging for the Advanced LIGO-Virgo detector network at design sensitivity to distinguish different source classes. We perform a Bayesian parameter-estimation analysis on a synthetic numerical-relativity signal in zero noise to study parameter biases. Finally, we reanalyze GW170817, with the hypothesis that it is a NSBH. We do not find evidence to distinguish the BNS and NSBH hypotheses; however, the posterior for the mass ratio is shifted to less equal masses under the NSBH hypothesis.
\end{abstract}

DOI: 10.1103/PhysRevD.102.043023

\section{INTRODUCTION}

In their first two observing runs (O1 and $\mathrm{O} 2)$, Advanced LIGO [1] and Advanced Virgo [2] have observed gravi-

Published by the American Physical Society under the terms of the Creative Commons Attribution 4.0 International license. Further distribution of this work must maintain attribution to the author(s) and the published article's title, journal citation, and DOI. Open access publication funded by the Max Planck Society. tational waves (GWs) from ten binary black holes (BBHs) and one binary neutron star (BNS), GW170817 [3]. Recently, in the third observing run (O3), a second BNS, GW190425, was discovered [4]. Other groups have reported additional $\mathrm{GW}$ observations analyzing the public 
data from the first two runs [5-7]. Neutron-star black holes (NSBHs) may be the next source class to be discovered. Given the lack of a detection in $\mathrm{O} 1$ and $\mathrm{O} 2$, the rate of $\mathrm{NSBHs}$ is uncertain. However, based on estimates from Ref. [8], the expected number of NSBH detections is $0_{-0}^{+19}$ in $\mathrm{O} 3$ and $1_{-1}^{+91}$ in $\mathrm{O} 4$ [9], where the central value is the median and the error bars give the $90 \%$ credible interval. As of this writing, in O3, the LIGO and Virgo Collaborations have published seven circulars via the Gamma-ray Coordinates Network (GCN) describing detection candidates for which the probability of the system being a NSBH is larger than $1 \%$ and for which the candidate has not been retracted [10-16]. Furthermore, GW data alone do not exclude the possibility that GW170817 is a NSBH [17-19], and it has also been suggested that GW190425 could be a NSBH $[20,21]$. Therefore it is timely to develop methods that can be used to study NSBHs in GW data.

NSBH binaries exhibit a rich phenomenology that is imprinted on the gravitational waveform (for a review see Ref. [22]). First, as is the case for BNS systems, finite-size effects cause a dephasing of the waveform relative to a $\mathrm{BBH}$ with the same masses and spins [23-26]. Additionally, the amplitude of NSBH waveforms can be affected by tidal forces [27]. For unequal mass ratios and slowly spinning $\mathrm{BHs}$, the amplitude of the waveform is well described by a $\mathrm{BBH}$ [28]. On the other hand, for near-equal mass ratios or for highly spinning BHs, depending on the NS equation of state (EOS), the NS can undergo tidal disruption, in which the star is ripped apart as it approaches the $\mathrm{BH}$ [27,29-32]. If the disruption takes place before the NS crosses the innermost stable circular orbit, then the material ejected from the NS can form a disk around the $\mathrm{BH}$ [33-35]. If so, starting at a characteristic (cutoff) frequency [27,36,37], the amplitude of the waveform is strongly suppressed, and the ringdown stage is reduced or even effaced. The details of this process contain information about the NS EOS. Additionally, NSBH mergers can be the progenitors of short gamma-ray bursts [38-43], and the disk around the remnant BH and dynamical ejecta can provide the engine for the kilonova signal [44,45], like the ones observed for GW170817 [3,46].

In order to take advantage of this potentially rich source of information, it is crucial to have a fast and accurate waveform model capturing effects due to relativistic matter, which can be used in analyzing GW data. Several approaches exist for describing finite-size effects in BNS systems. Tidal corrections [23-25,47,48] have been incorporated in the effective-one-body (EOB) formalism [49-51] in Refs. [52-57]. References [58,59] developed a flexible technique that starts from a point-mass $\mathrm{BBH}$ baseline waveform and applies tidal-phase modifications by fitting a Padé-resummed post-Newtonian (PN)-based ansatz to the phasing extracted from numerical-relativity (NR) simulations (henceforth, we refer to this as the NRTidal approach). These corrections have been applied to $\mathrm{BBH}$ baselines produced within the EOBNR framework [60] and within the inspiral-merger-ringdown phenomenological (IMRPhenom) approach [61,62].

There have been several previous works constructing NSBH waveforms. An aligned-spin NSBH waveform model was developed in Refs. [63,64], but it covered a limited range of mass ratios. In Ref. [65], this waveform model was used in parameter and population studies in conjunction with a former version of the EOBNR BBH baseline [66]. A NSBH model called PhenomNSBH, which was constructed using a similar approach to modeling NSBHs as the one discussed in this paper but developed within the IMRPhenom approach, was recently put forward in Ref. [67]. This model uses the method of Ref. [68] to describe tidal disruption of the amplitude and uses the tidalphase corrections from Ref. [59].

In this work we develop a frequency-domain model for the dominant, quadrupolar multipole of GWs emitted by aligned-spin NSBH systems. Together with the recent waveform model of Ref. [67], these are the first NSBH models covering a wide range of mass ratios and spin that can be used to analyze GW data. In this paper, we refer to our model as SEOBNR_NSBH, which has already been implemented in the LIGO algorithms library (LAL) [69]. In Table I we provide a dictionary between the names we use in this work and the name as implemented in LAL. The amplitude is based on an EOBNR BBH baseline model that we refer to as SEOBNR_BBH [60]. We apply corrections inspired by Pannarale et al. [68] to account for tidal disruption. We have adapted the corrections of Ref. [68], originally developed for a former version of the IMRPhenom BBH model [70], for use with EOBNR waveforms [60], augmented with reduced-order modeling (ROM) [71,72] to enhance the speed. Differently from Ref. [67], which uses the fit from Pannarale et al. [68], here we have performed a fit incorporating results from the new

TABLE I. Dictionary relating the names we use in this paper for several waveforms from the SEOBNR family and the corresponding names of the waveforms implemented in the LIGO algorithms library (LAL). The second and third waveforms use tidal effects within the NRTidal approach.

\begin{tabular}{lcr}
\hline \hline Name in this paper & LAL name & Ref. \\
\hline SEOBNR_BBH & SEOBNRv4_ROM & {$[60]$} \\
SEOBNR_BNS & SEOBNRv4_ROM_NRTidalv2 & {$[59]$} \\
SEOBNR_NSBH & SEOBNRv4_ROM_NRTidalv2_NSBH & This paper \\
\hline \hline
\end{tabular}


NSBH simulations at our disposal as described in Sec. II B. While the publicly available Simulating eXtreme Spacetimes (SXS) simulations were not used for calibration of Ref. [67], these waveforms were used for validation and good agreement was found. The phase is computed by applying tidal corrections to the EOBNR BBH baseline [60] using the NRTidal approach, as in SEOBNR_BNS [59]. As shown in Ref. [73], even though the tidal corrections from SEOBNR_BNS were derived from BNS simulations, they give good agreement with NSBH simulations.

The rest of this paper is organized as follows. In Sec. II, we describe the construction of the waveform model. We review properties of NSBH systems in Sec. II A, summarize the NR waveforms that we use in Sec. II B, give an outline of the waveform model in Sec. II C, and summarize the procedure used to calibrate the amplitude correction, assess their accuracy by computing the unfaithfulness, and compare the NSBH waveforms to NR simulations in Sec. II D. Then, we discuss the regime of validity in Sec. IIE. Next, we apply the waveform model to several data-analysis problems. First, in Sec. III A we estimate when the Advanced LIGO-Virgo detector network at design sensitivity can distinguish NSBH and $\mathrm{BBH}$, and $\mathrm{NSBH}$ and BNS, systems. Then, in Sec. III B we perform parameterestimation Bayesian analysis on an NR waveform, hybridized to an analytical waveform at low frequency, and show the differences between recovering this waveform with a BBH and NSBH model. Finally, we reanalyze GW170817 under the hypothesis that it is a NSBH in Sec. III C. We conclude in Sec. IV by summarizing the main points and lay out directions for future improvements. Finally, in the Appendix, we give explicit expressions defining the waveform model.

We work in units with $G=c=1$. The symbol $M_{\odot}$ refers to the mass of the Sun.

\section{CONSTRUCTING THE NSBH WAVEFORM MODEL}

\section{A. NSBH binary properties}

We begin by providing a brief description of the final stages of a NSBH coalescence, identifying the main features of the process and the physical properties of the remnant $\mathrm{BH}$. Here, we mainly follow the discussion in Refs. [22,34,68].

The two bodies spiral in due to the loss of energy from the emission of GWs. If the NS approaches close enough to the $\mathrm{BH}$, tidal forces exerted by the $\mathrm{BH}$ on the NS can overcome the self-gravity of the NS, causing the star to lose mass. This process is called mass shedding. This in turn often leads to tidal disruption, in which the NS is completely torn apart by the strong gravitational field of the BH. Let us denote with $r_{\text {tide }}$ the separation of the binary at which mass shedding begins. To understand the fate of the NS and the characteristics of the GW signal emitted during the last stages of inspiral, plunge and merger, we compare $r_{\text {tide }}$ to the location of the innermost-stable circular orbit (ISCO) (which marks the beginning of the plunge). If $r_{\text {tide }}<r_{\text {ISCO }}$, the NS is swallowed by the $\mathrm{BH}$, without loss of material. By contrast, if $r_{\text {tide }}>r_{\text {ISCO }}$, mass is ejected from the NS before it plunges. If the NS is far away from the ISCO when it is disrupted, matter may form an accretion disk (torus) around the $\mathrm{BH}$ after merger. It has been shown (e.g., see Refs. [27,30,34,35] and also below) that the disruption affects the GW signal for NSBH binaries with either nearly equal masses or large $\mathrm{BH}$ spins aligned with the orbital angular momentum, because for those systems the condition $r_{\text {tide }}<r_{\text {ISCO }}$ is satisfied. In Fig. 1, we show an illustration of the effect of tidal disruption on the GW waveform for an example NR hybrid with mass ratio 1.5 .

Let us now estimate the radial separation at which mass shedding occurs, $r_{\text {tide }}$, by imposing that the tidal force from the BH balances the self-gravity of the NS. As described in Ref. [34], in the Newtonian limit, $r_{\text {tide }}$ can be estimated as $r_{\text {tide }} \approx \xi_{\text {Newt }} R_{\mathrm{NS}}$, where $\xi_{\text {Newt }}=(3 Q)^{1 / 3}, R_{\mathrm{NS}}$ is the NS radius in isolation, $Q \equiv M_{\mathrm{BH}} / M_{\mathrm{NS}}$ is the mass ratio, $M_{\mathrm{BH}}$ is the mass of the $\mathrm{BH}$, and $M_{\mathrm{NS}}$ is the mass of the NS. The factor of 3 is an estimate obtained by matching with NR simulations. This estimate can be improved by accounting for relativistic effects due to the large compactness of the $\mathrm{NS}, C_{\mathrm{NS}} \equiv M_{\mathrm{NS}} / R_{\mathrm{NS}}$ [34]. First, $r_{\text {tide }}$ is reduced by a factor $\left(1-2 C_{\mathrm{NS}}\right)$ relative to the Newtonian estimate; this factor enforces the absence of tidal disruption in the $\mathrm{BH}$ limit $C_{\mathrm{NS}} \rightarrow 1 / 2$. Second, point-mass motion in the Kerr metric leads to a correction factor $\xi$ which differs from the Newtonian estimate $[34,74]$. Combining these effects, we have

$$
r_{\text {tide }}=\xi\left(1-2 C_{\mathrm{NS}}\right) R_{\mathrm{NS}} .
$$

The relativistic correction parameter $\xi$ is determined by solving the algebraic equation (we take the largest positive root of this equation)

$$
\left(\frac{\xi}{\xi_{\mathrm{Newt}}}\right)^{3}=\frac{\xi^{2}-2 Q C_{\mathrm{NS}} \xi+Q^{2} C_{\mathrm{NS}}^{2} \chi_{\mathrm{BH}}^{2}}{\xi^{2}-3 Q C_{\mathrm{NS}} \xi+2 \chi_{\mathrm{BH}} \sqrt{Q^{3} C_{\mathrm{NS}}^{3} \xi}}
$$

where $\chi_{\mathrm{BH}}$ is the spin of the $\mathrm{BH}$. We can associate to the tidal-disruption separation a frequency, which is more useful in the context of modeling the gravitational waveform, as follows:

$$
f_{\text {tide }}=\frac{1}{\pi\left(\chi_{\mathrm{BH}} M_{\mathrm{BH}}+\sqrt{r_{\text {tide }}^{3} / M_{\mathrm{BH}}}\right)},
$$




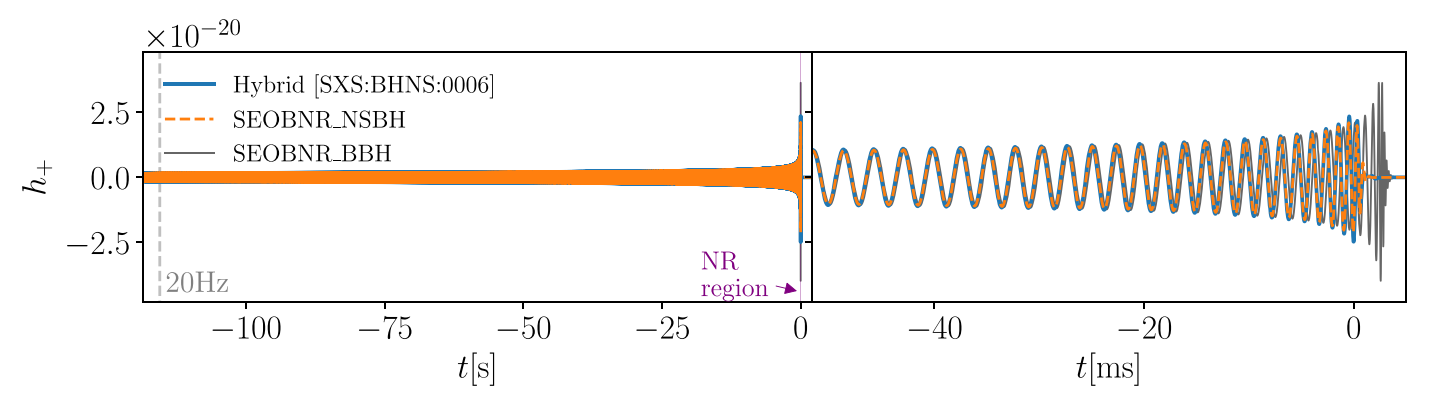

FIG. 1. We compare an NR-hybrid waveform, which is constructed by stitching together the SXS:BHNS:0006 $\left(M_{\mathrm{NS}}=1.4 M_{\odot}\right.$, $\left.M_{\mathrm{BH}}=2.1 M_{\odot}, \Lambda_{\mathrm{NS}}=791\right)$ and SEOBNR_BNS $\left(m_{1}=1.4 M_{\odot}, m_{2}=2.1 M_{\odot}, \Lambda_{1}=0, \Lambda_{2}=791\right)$ waveforms, with the SEOBNR_NSBH $\left(M_{\mathrm{NS}}=1.4 M_{\odot}, M_{\mathrm{BH}}=2.1 M_{\odot}, \Lambda_{\mathrm{NS}}=791\right)$ and SEOBNR_BBH $\left(m_{1}=1.4 M_{\odot}, m_{2}=2.1 M_{\odot}, \Lambda_{1}=\Lambda_{2}=0\right)$ waveforms. The strain is produced by a source at a distance of $1 \mathrm{Mpc}$. The definition of the tidal parameter $\Lambda_{\mathrm{NS}}$ is given in Eq. (4). In the left panel, we show the waveforms during the long inspiral and mark $20 \mathrm{~Hz}$, which is the lower frequency typically used for LIGO-Virgo parameter-estimation analyses. We also indicate the region toward merger where the NR data are available. In the right panel, we enlarge the last stages of the inspiral and merger. Due to tides, the hybrid waveform and SEOBNR_NSBH have a faster inspiral and end earlier than the corresponding point-mass SEOBNR_BBH waveform. Furthermore, because of tidal disruption, the hybrid and SEOBNR_NSBH waveforms have no ringdown phase but end abruptly when the NS gets disrupted. Overall, we find very good agreement between SEOBNR_NSBH and NR-hybrid waveforms throughout the entire coalescence.

which is obtained from the (circular orbit) relation between radial separation and (angular) orbital frequency in the Kerr geometry. ${ }^{1}$

The NS compactness $C_{\mathrm{NS}}$, which depends on the NS EOS, enters the expression for $r_{\text {tide }}$. In order to avoid making an assumption about the EOS, it is more convenient to work in terms of the dimensionless tidal-deformability parameter $\Lambda_{\mathrm{NS}}$, which relates the quadrupole moment of the NS to the tidal field of the companion. The tidal parameter is determined by the compactness of the NS and the tidal Love number $k_{2}$ as follows:

$$
\Lambda_{\mathrm{NS}}=\frac{2}{3} \frac{k_{2}}{C_{\mathrm{NS}}^{5}} .
$$

We take the tidal parameter of the $\mathrm{BH}$ to be zero. The tidal parameter of nonspinning BHs was shown to be zero in Ref. [75]. We can relate $\Lambda_{\mathrm{NS}}$ and $C_{\mathrm{NS}}$ in an equation-ofstate-independent way with the $\Lambda_{\mathrm{NS}}-C_{\mathrm{NS}}$ relation [76]

$$
C_{\mathrm{NS}}=\sum_{k=0}^{2} a_{k}\left(\ln \Lambda_{\mathrm{NS}}\right)^{k},
$$

with $a_{0}=0.360, a_{1}=-0.0355$, and $a_{2}=0.000705$. In order to achieve continuity with $\mathrm{BBH}$ waveforms in the

\footnotetext{
${ }^{1}$ Note that in [68], the formula for $f_{\text {tide }}$ is written in terms of the final, rather than initial, $\mathrm{BH}$ mass and spin. In LAL, SEOBNR_NSBH is implemented with the final BH properties. We became aware of this point during a late stage of this work. The fits in this work were done self-consistently using the final $\mathrm{BH}$ properties. We have checked that when we replace $M_{f}$ and $\chi_{f}$ by $M_{\mathrm{BH}}$ and $\chi_{\mathrm{BH}}$ in the expression for $f_{\text {tide }}$, mismatches with SEOBNR_NSBH are $\mathcal{O}\left(10^{-4}\right)$ or less across parameter space. We thank the internal LIGO review team for bringing this to our attention.
}

limit $\Lambda_{\mathrm{NS}} \rightarrow 0$, for $\Lambda_{\mathrm{NS}} \leq 1$, we replace the $\Lambda_{\mathrm{NS}}-C$ relation with a cubic polynomial which interpolates from $\Lambda_{\mathrm{NS}}=1$ to $C_{\mathrm{NS}}=1 / 2$ at $\Lambda_{\mathrm{NS}}=0$, and it is continuous and once differentiable at $\Lambda_{\mathrm{NS}}=1$. The universal relations are also used in Ref. [67].

The matter ejected from the NS, during tidal disruption, can remain bound, forming a disk (torus) around the remnant $\mathrm{BH}$. The mass of this remnant torus, $M_{b \text {,torus }}$, can be determined in terms of the baryonic mass of the NS using fits from Ref. [34] (see also more recent simulations performed in Ref. [77]):

$$
\frac{M_{b, \text { torus }}}{M_{b, \mathrm{NS}}}=\max \left(\frac{0.296 r_{\mathrm{tide}}-0.171 r_{\mathrm{ISCO}}}{R_{\mathrm{NS}}}, 0\right),
$$

where the ISCO radius ( $\left.r_{\mathrm{ISCO}}\right)$ in the Kerr spacetime is given by

$$
\begin{aligned}
& r_{\mathrm{ISCO}}=3+Z_{2} \mp \sqrt{\left(3-Z_{1}\right)\left(3+Z_{1}+2 Z_{2}\right)}, \\
& Z_{1}=1+\left(1-\chi_{\mathrm{BH}}^{2}\right)^{1 / 3}\left[\left(1+\chi_{\mathrm{BH}}\right)^{1 / 3}+\left(1-\chi_{\mathrm{BH}}\right)^{1 / 3}\right], \\
& Z_{2}=\sqrt{3 \chi_{\mathrm{BH}}^{2}+Z_{1}^{2}},
\end{aligned}
$$

where the $\mp$ sign holds for prograde (retrograde) orbits.

As mentioned above, the onset of mass shedding occurs when the objects approach within a distance $r_{\text {tide }}$ before the NS cross the ISCO. However, the ISCO does not introduce a definite feature in the gravitational waveform. In order to identify the onset of tidal disruption with a definite feature in an NR waveform, in our model we compare $f_{\text {tide }}$ to the ringdown frequency of the final $\mathrm{BH}, f_{\mathrm{RD}}$, which is the frequency of least-damped quasinormal mode of the final $\mathrm{BH}$. The ringdown frequency can be computed from the 
final mass and spin using fitting formulas from Ref. [78]. To obtain the final mass and spin from the initial parameters of the binary, we use the fits performed by Ref. [79], which account for the ejected mass.

\section{B. Numerical-relativity waveforms}

In this section we briefly describe the NR data used to construct and validate the model. The SXS Collaboration has publicly released data from seven simulations described in Refs. [73,80], which were produced using the spectral Einstein code (SpEC); see Ref. [81]. The hyrodynamical part of the code is described in Refs. [82,83]. These configurations do not contain spinning BHs but do include mergers with and without tidal disruption. These simulations use an ideal gas EOS with polytropic index $\Gamma=2$, except for the mass ratio 3 simulation SXS:BHNS:0003, which uses a piecewise polytropic ansatz calibrated to the H1 EOS; see Ref. [84]. We refer the reader to Ref. [73] for further explanation. For five of these simulations the NS spin is zero, and we use these simulations to fit the model as described in Sec. II D. We use the other two simulations for verification. Additionally, SpEC has simulated nine systems with large $\mathrm{BH}$ spin in Ref. [85], using the more advanced temperature- and composition-dependent LS220 EOS [86], which we also use to fit our waveform model. Finally, we validate our NSBH model also against two new SXS waveforms, Q3S9 and Q4S9, which are highly accurate simulations describing disruptive mergers with large BH spin. These simulations were also performed using the $\Gamma=2$ EOS. We give the parameters of all SXS waveforms used here in Table II.

In fitting the model, we also use 134 simulations of irrotational NSs performed with the SACRA code [87], which were presented in Refs. [27,30]. These simulations span the mass ratios $Q=\{2,3,4,5\}$, BH spins $\chi_{\mathrm{BH}}=$ $\{-0.5,0,0.25,0.5,0.75\}$, and a range of piecewise polytropic EOS. The parameters for all of the waveforms and the EOS used are given Table II of Ref. [64]. Whereas the large number of SACRA waveforms lets us probe a wide parameter range, these waveforms are shorter and of lower accuracy than the publicly available SpEC waveforms as well as Q3S9 and Q4S9, due to finite numerical resolution and non-negligible eccentricity in the initial data. We note that these simulations predate the public SXS simulations by a number of years.

\section{Parameterization of the NSBH waveform model}

We limit the waveform modeling to the dominant quadrupolar multipole, notably the modes $\ell=2, m= \pm 2$ in the -2 spin-weighted spherical harmonic decomposition of the gravitational polarizations $h_{+, x}$, and to aligned-spin NSBHs. In the frequency domain, we can write the waveform as

$$
h(f)=A(f) e^{i \phi(f)}
$$

Henceforth, we focus on the dependence of the amplitude $A(f)$ and phase $\phi(f)$ on the intrinsic parameters of the

TABLE II. Parameters for the SXS NSBH waveforms used in this work. The simulations above the horizontal line were used to fit the NSBH model, and the simulations below the line are used for validation. Parameters for the other waveforms that we employ to fit the model were produced by the SACRA code and are given in Table II of Ref. [64]. We also report the number of GW cycles, $N_{\mathrm{GW}}$, computed up to the peak of the dominant GW mode. Finally we display the residual eccentricity $e_{\text {res }}$.

\begin{tabular}{llllllllr}
\hline \hline Label & $\frac{M_{\mathrm{BH}}}{M_{\odot}}$ & $\frac{M_{\mathrm{NS}}}{M_{\odot}}$ & $Q$ & $\chi_{\mathrm{BH}}$ & $\chi_{\mathrm{NS}}$ & $\Lambda_{\mathrm{NS}}$ & $N_{\mathrm{GW}}$ & $e_{\text {res }}$ \\
\hline SXS:BHNS:0001 & 8.4 & 1.4 & 6 & 0 & 0 & 526 & 25.3 & $<1.3 \times 10^{-3}$ \\
SXS:BHNS:0002 & 2.8 & 1.4 & 2 & 0 & 0 & 791 & 26.1 & $<5 \times 10^{-4}$ \\
SXS:BHNS:0003 & 4.05 & 1.35 & 3 & 0 & 0 & 624 & 12.3 & $7.9 \times 10^{-3}$ \\
SXS:BHNS:0004 & 1.4 & 1.4 & 1 & 0 & 0 & 791 & 24.5 & $<6.0 \times 10^{-5}$ \\
SXS:BHNS:0006 & 2.1 & 1.4 & 1.5 & 0 & 0 & 791 & 33.2 & $<2.7 \times 10^{-4}$ \\
M12-7-S8-LS220 & 7 & 1.2 & 5.8 & 0.8 & 0 & 1439 & 17.8 & $2.7 \times 10^{-2}$ \\
M12-7-S9-LS220 & 7 & 1.2 & 5.8 & 0.9 & 0 & 1439 & 18.9 & $2.6 \times 10^{-2}$ \\
M12-10-S8-LS220 & 10 & 1.2 & 8.3 & 0.8 & 0 & 1439 & 20.3 & $3.1 \times 10^{-2}$ \\
M12-10-S9-LS220 & 10 & 1.2 & 8.3 & 0.9 & 0 & 1439 & 22.1 & $3.3 \times 10^{-2}$ \\
M14-7-S7-LS220 & 7 & 1.4 & 5 & 0.7 & 0 & 536 & 10.6 & $3.9 \times 10^{-2}$ \\
M14-7-S8-LS220 & 7 & 1.4 & 5 & 0.8 & 0 & 536 & 11.7 & $3.7 \times 10^{-2}$ \\
M14-7-S9-LS220 & 7 & 1.4 & 5 & 0.9 & 0 & 536 & 12.5 & $3.7 \times 10^{-2}$ \\
M14-10-S8-LS220 & 10 & 1.4 & 7.1 & 0.8 & 0 & 536 & 15.1 & $4.2 \times 10^{-2}$ \\
M14-10-S9-LS220 & 10 & 1.4 & 7.1 & 0.9 & 0 & 536 & 16.8 & $4.3 \times 10^{-2}$ \\
\hline SXS:BHNS:0005 & 1.4 & 1.4 & 1 & 0 & -0.2 & 791 & 21.6 & $5.0 \times 10^{-4}$ \\
SXS:BHNS:0007 & 2.8 & 1.4 & 2 & 0 & -0.2 & 791 & 24.7 & $<4.7 \times 10^{-4}$ \\
Q3S9 & 4.2 & 1.4 & 3 & 0.9 & 0 & 791 & 26.5 & $5.4 \times 10^{-4}$ \\
Q4S9 & 5.6 & 1.4 & 4 & 0.9 & 0 & 791 & 31.4 & $1.7 \times 10^{-3}$ \\
\hline \hline
\end{tabular}


binary, $\vec{\theta}=\left\{M_{\mathrm{BH}}, M_{\mathrm{NS}}, \chi_{\mathrm{BH}}, \chi_{\mathrm{NS}}, \Lambda_{\mathrm{NS}}\right\}$, where we indicate with $\chi_{\mathrm{BH}}$ and $\chi_{\mathrm{NS}}$ the (dimensionless) components of the spin aligned with the orbital angular momentum, for the $\mathrm{BH}$ and NS, respectively.

To compute the GW phase $\phi(f)$, we use the point-mass baseline SEOBNR_BBH model and apply tidal corrections from the NRTidal framework, as in Ref. [59]. As shown in Ref. [73] (and as we verify in Figs. 5 and 6), applying NRTidal corrections gives a reasonable approximation of the phase, until the last few cycles.

In order to model the amplitude $A(f)$, we start with the BNS model SEOBNR_BNS as a baseline. Since this model includes tapering beyond the BNS merger frequency [88], we first remove this tapering. This is necessary since the tapering depends on the tidal parameters of both objects, $\Lambda_{1}$ and $\Lambda_{2}$, and does not vanish as $\Lambda_{1} \rightarrow 0$. We note that this means that the $\Lambda_{1} \rightarrow 0$ limit of SEOBNR_BNS does not correctly describe the amplitude of a NSBH system.

We then apply a correction to the amplitude that describes the tidal-disruption effects discussed in the previous section. More precisely, we relate the amplitude of SEOBNR_NSBH, $A(f)$, to the amplitude of SEOBNR_BNS with no tapering or tidal amplitude corrections applied, $A_{\mathrm{NRT} \text {-notaper }}(f)$, via

$$
A(f)=w_{\text {corr }}(f) A_{\text {NRT-notaper }}(f),
$$

where the correction function $w_{\text {corr }}$ is given by

$$
w_{\text {corr }}(f)=w^{-}\left(f ; f_{0}, \sigma\right)+\epsilon w^{+}\left(f ; f_{0}, \sigma\right)
$$

and $w^{ \pm}\left(f ; f_{0}, \sigma\right)$ are the hyperbolic-tangent window functions

$$
w^{ \pm}\left(f ; f_{0}, \sigma\right)=\frac{1}{2}\left[1 \pm \tanh \left(\frac{4\left(f-f_{0}\right)}{\sigma}\right)\right] .
$$

We illustrate the behavior of $w_{\text {corr }}$ in Fig. 3. When $\epsilon=0$, $w_{\text {corr }}(f)$ cuts off the amplitude before the end expected for a $\mathrm{BBH}$ system with the same masses and spins of the NSBH and therefore describes tidal disruption. When $\epsilon>0$, the final part of the inspiral and the postmerger signal are still present but are suppressed relative to the BBH case. The parameters $f_{0}, \sigma$, and $\epsilon$, which determine the precise nature of these corrections, are determined by comparing with NR simulations.

Following Ref. [68], we classify the waveforms into four cases: nondisruptive, disruptive, mildly disruptive without torus remnant, and mildly disruptive with torus remnant, depending on the intrinsic parameters of the system. To determine the three parameters $\left\{f_{0}, \sigma_{0}, \epsilon\right\}$ in Eq. (9), we adapt the amplitude model of Ref. [68], which was developed for a different BBH baseline, to the SEOBNR_BBH model. We then calibrate the parameters of this model, using the method described in Sec. II D.

\section{Nondisruptive mergers: $f_{\mathrm{RD}}<f_{\text {tide }}, M_{b \text {,torus }}=0$}

When the tidal frequency is larger than the ringdown frequency of the final $\mathrm{BH}$, the NS reaches the ISCO before crossing $r_{\text {tide }}$. In this case the NS remains intact as it plunges, but with a slightly suppressed amplitude of the ringdown. The waveform is very similar to a $\mathrm{BBH}$. To describe this, we use $f_{0}=f_{\mathrm{ND}}, \sigma_{0}=\sigma_{\mathrm{ND}}$, and $\epsilon=\epsilon_{\mathrm{ND}}$, where ND stands for nondisruptive, and

$w_{\mathrm{ND}}(f)=w^{-}\left(f ; f_{\mathrm{ND}}, \sigma_{\mathrm{ND}}\right)+\epsilon_{\mathrm{ND}} w^{+}\left(f ; f_{\mathrm{ND}}, \sigma_{\mathrm{ND}}\right)$.

The explicit expressions relating $f_{\mathrm{ND}}, \sigma_{\mathrm{ND}}$, and $\epsilon_{\mathrm{ND}}$ to the intrinsic parameters of the binary are given in the Appendix.

\section{Disruptive mergers: $f_{\mathrm{RD}}>f_{\text {tide }}, M_{b, \text { torus }}>0$}

In this case, tidal disruption occurs and a remnant torus of matter forms. For such systems, the typical merger and ringdown stages present for BBHs are exponentially suppressed. To model this case, we set $\epsilon=0$, so that the waveform decays above a frequency $f_{\mathrm{D}}$ with width $\sigma_{\mathrm{D}}$. This leads to the expression

$$
w_{\mathrm{D}}(f)=w^{-}\left(f ; f_{\mathrm{D}}, \sigma_{\mathrm{D}}\right) .
$$

The precise definition is given in the Appendix.

\section{Mildly disruptive mergers with no torus remnant: $f_{\mathrm{RD}}>f_{\text {tide }}, M_{b, \text { torus }}=0$}

In this case, the NS undergoes mass shedding, but no torus forms around the remnant $\mathrm{BH}$. We combine the information from the nondisruptive and disruptive cases to determine the cutoff frequency and the width of the tapering. We set $f_{0}=\left(1-Q^{-1}\right) f_{\mathrm{ND}}+Q^{-1} f_{\text {tide }}$ and $\sigma_{0}=\left(\sigma_{\mathrm{D}}+\sigma_{\mathrm{ND}}\right) / 2, \epsilon=0$.

\section{Mildly disruptive mergers with torus remnant: $f_{\text {RD }}<f_{\text {tide }}, M_{b, \text { torus }}>0$}

In this scenario the tidal frequency is above the ringdown frequency, but there is a remnant disk of matter around the BH. As discussed, for example, in Ref. [22], this scenario occurs at large $\mathrm{BH}$ spins and represents the case in which the NS is disrupted before crossing the ISCO, but the size of the tidally disrupted material in the vicinity of the $\mathrm{BH}$ is smaller than the BH surface area. Thus, in this case, although a remnant disk eventually forms, the matter does not distribute uniformly around the $\mathrm{BH}$ quickly enough to cancel coherently or suppress the $\mathrm{BH}$ oscillations. As a consequence, the ending part of the NSBH waveform contains a ringdown signal. In this case, we again combine information from cases 1 and 2 and fix $f_{0}=f_{\mathrm{D}}, \sigma_{0}=\sigma_{\mathrm{ND}}$, and $\epsilon=\epsilon_{\mathrm{ND}}$. 
In Fig. 2, we show the regions of these different parameter spaces, along with relevant NR simulations from the SACRA and SpEC codes.

\section{Fitting procedure}

The amplitude correction described in the previous section has 20 free parameters, which we denote with the vector $\vec{\lambda}$. The definition of these parameters is given in the Appendix. We fix the coefficients in $\vec{\lambda}$ by requiring that the SEOBNR_NSBH waveforms agree, as much as possible, with the SpEC and SACRA waveforms described in Sec. II B.

For a given NR waveform indexed by I, let us denote the Fourier-domain amplitude of the dominant mode by $A_{\mathrm{I}}^{\mathrm{NR}}(f ; \vec{\theta})$. Given the intrinsic parameters of the binary, $\vec{\theta}$, and a set of fit parameters $\vec{\lambda}$, we compute the following quantity $\Delta_{\mathrm{I}}(\vec{\lambda})^{2}$ :
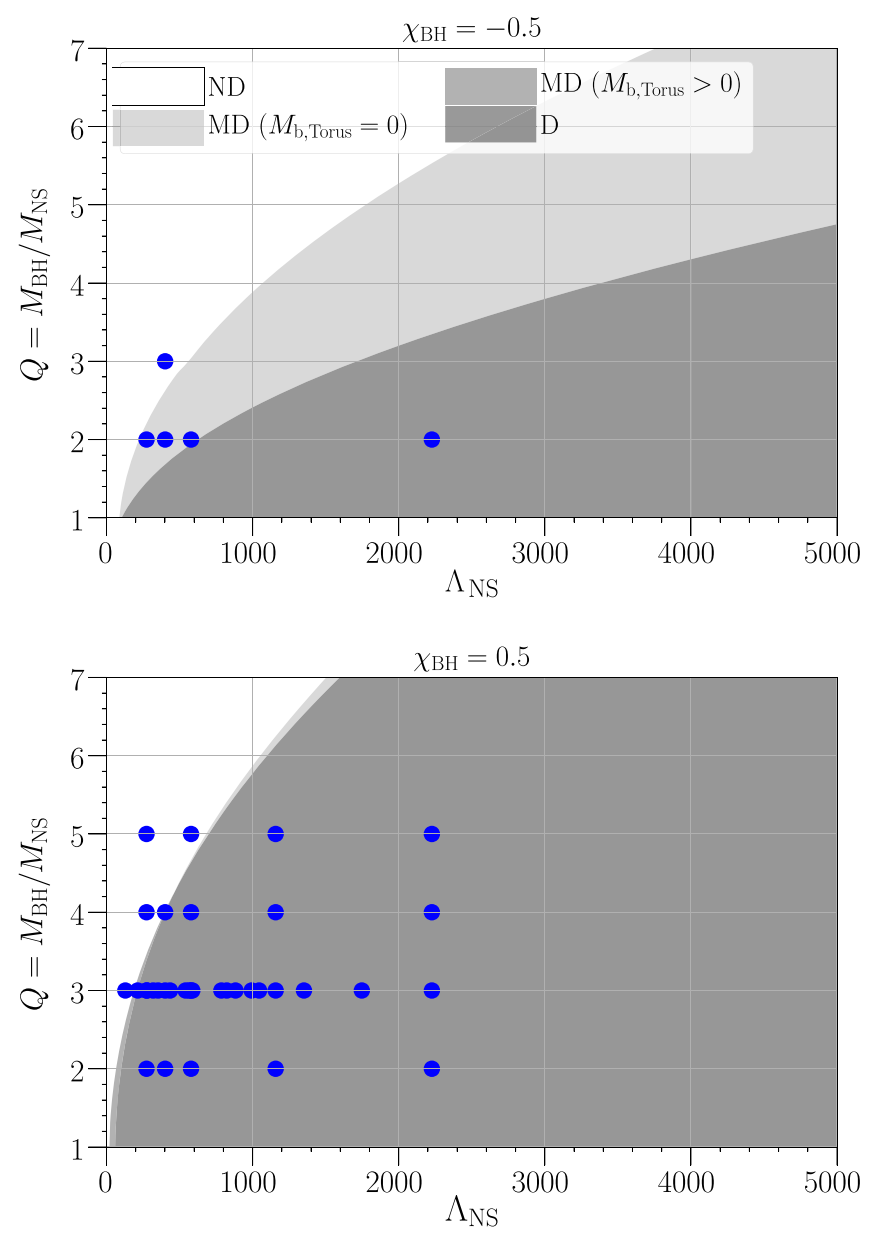

$$
\Delta_{\mathrm{I}}(\vec{\lambda})^{2}=\int_{f_{\min }}^{f_{\text {cut }}} \mathrm{d} f \frac{\left[A(f ; \vec{\theta}, \vec{\lambda})-A_{\mathrm{I}}^{\mathrm{NR}}(f ; \vec{\theta})\right]^{2}}{\sigma_{\mathrm{I}}(f)^{2}},
$$

to estimate the difference between the frequency-domain amplitude of the model $A(f)$ and of the NR simulation $A_{\mathrm{I}}^{\mathrm{NR}}(f)$. We choose the lower bound of the integral $f_{\min }$ to be the frequency at which $A_{\mathrm{I}}^{\mathrm{NR}}(f)$ falls to $90 \%$ of its initial (lowest-frequency) value; this is a low enough frequency to ensure $w_{\text {corr }}\left(f_{\min }\right) \approx 1$ while avoiding possible contamination from eccentricity in the initial data. For the upper frequency, we take a definition inspired by the cutoff frequency given in Ref. [37]. First, we define $f_{\max }$ to be the frequency at which $f^{2} A(f)$ takes its maximum value. Then we define $f_{\text {cut }}$ to be the frequency (larger than $f_{\max }$ ) which satisfies

$$
f_{\text {cut }} A_{\mathrm{I}}^{\mathrm{NR}}\left(f_{\text {cut }}\right)=0.1 f_{\max } A_{\mathrm{I}}^{\mathrm{NR}}\left(f_{\max }\right) .
$$
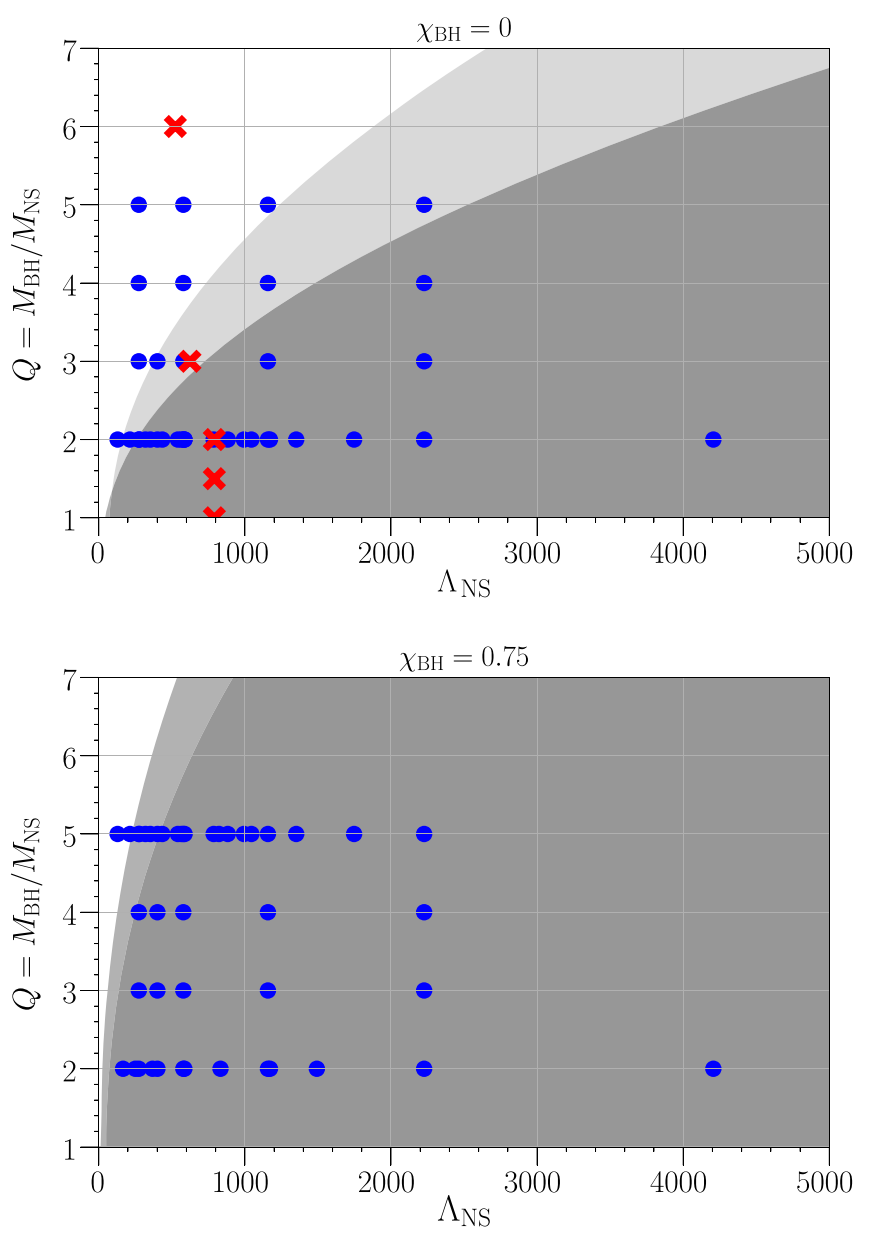

FIG. 2. Representative parameter space region in the $Q-\Lambda_{\mathrm{NS}}$ plane for different values of the $\mathrm{BH}$ spin $\chi_{\mathrm{BH}}$. We show the regions for the different classes of NSBHs, using the model described in the main text: white regions represent nondisruptive mergers (ND), light gray regions represent mildly disruptive mergers without a torus remnant ( $\mathrm{MD}, M_{b, \text { torus }} \neq 0$ ), the medium gray shade visible in the $\chi_{\mathrm{BH}}=0.75$ figure represents mildly disruptive mergers with a torus remnant $\left(\mathrm{MD}, M_{b, \text { torus }}=0\right.$ ), and the dark gray region marks disruptive mergers with a torus remnant (D). We also mark the parameter values of (some of) the NR simulations used. Dots (crosses) represent simulations produced by the SACRA (SpEC) code. Not shown are 12 SACRA waveforms with $\chi_{\mathrm{BH}}=0.25$, two SpEC waveforms with NS spin different from zero, and 11 SpEC waveforms with $\chi_{\mathrm{BH}}=\{0.7,0.8,0.9\}$. 


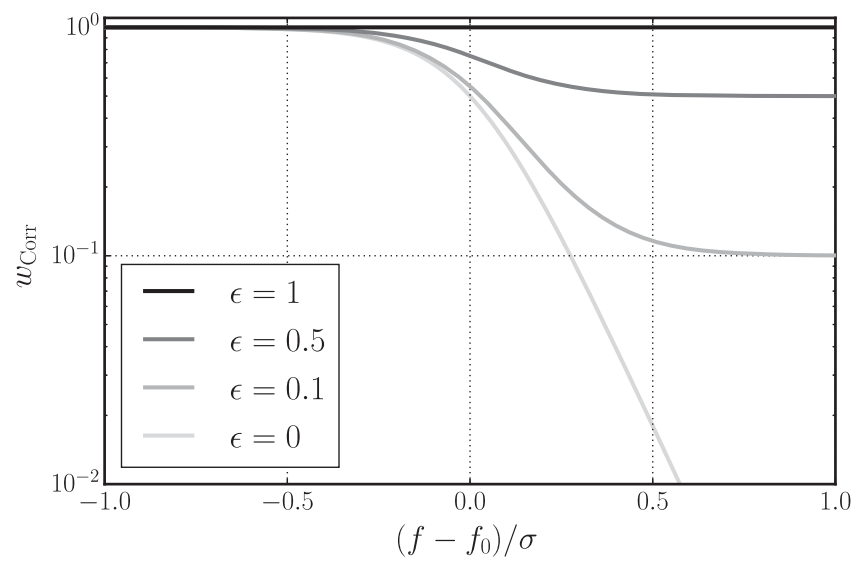

FIG. 3. We illustrate the behavior of the function $w_{\text {corr }}(f)$ in Eq. (9), which modifies the amplitude of the waveform with respect to the one of a $\mathrm{BNS}$ and $\mathrm{BBH}$. The central frequency $f_{0}$ determines the frequency at which the amplitude is tapered; the width $\sigma$ determines the range of frequencies over which the tapering takes place; the parameter $\epsilon$ keeps the postmerger signal at a suppressed level, if it is nonzero.

This frequency is larger than the ringdown frequency for nondisruptive mergers. For disruptive mergers, $f_{\text {cut }}$ gives a characteristic frequency at which the frequency-domain amplitude has been suppressed. For the error function in Eq. (14), we consider a constant relative error at each frequency given by $\sigma_{\mathrm{I}}(f)=k_{\mathrm{I}} A_{\mathrm{I}}(f)$. We use $k_{\mathrm{I}}=1$ for the SACRA waveforms, and $k_{\mathrm{I}}=0.1$ for the SpEC waveforms, to account for the difference in length and accuracy in the waveforms. We then compute a global error, for a given subset $\mathcal{S}$ of the NR waveforms, by summing $\Delta_{\text {I }}^{2}$ over all NR waveforms in $\mathcal{S}$ :

$$
\Delta^{2}(\vec{\lambda})=\sum_{\mathrm{I} \in \mathcal{S}} \Delta_{\mathrm{I}}^{2}(\vec{\lambda})
$$

We minimize $\Delta^{2}(\vec{\lambda})$ with respect to $\vec{\lambda}$ using the NelderMead algorithm [89]. We first use the parameter values from Ref. [68] as an initial guess and minimize the error over the parameters of the nondisruptive and disruptive window functions separately. We then use the results of this fit as an initial guess for a global fit, including all of the available waveforms in $\mathcal{S}$. The final results of this global fit are used to define the model, and the numerical values are given in Table VII in the Appendix.

We now turn to a quantitative assessment of the model's performance by comparing against NR simulations. We additionally compare with the recently developed PhenomNSBH model of Ref. [67], in order to understand the performance of the two approximants relative to NR and to each other. To this end, we employ the faithfulness function given in Ref. [90], which is commonly used in LIGO and Virgo data analysis to assess the agreement of two waveforms, e.g., the template $\tau$ and the signal $s$. Let us first introduce the inner product between two waveforms $a$ and $b[91,92]$ :

$$
\langle a \mid b\rangle \equiv 4 \operatorname{Re} \int \mathrm{d} f \frac{a^{*}(f) b(f)}{S_{n}(f)},
$$

where a star denotes the complex conjugate and $S_{n}(f)$ is the one-sided, power spectral density (PSD) of the detector noise. Here, we use the Advanced LIGO design sensitivity PSD as given in Ref. [93]. We compute the faithfulness $\mathcal{F}$ by maximizing the normalized inner product (or overlap) over the coalescence time $t_{c}$, the initial phase $\phi_{0 \tau}$ of the template $\tau$, and setting the phase of the signal $\phi_{0 s}$ to zero at merger, while fixing the same parameters $\vec{\theta}$ for the template and the signal, that is,

$$
\mathcal{F} \equiv \max _{t_{c}, \phi_{0 \tau}}\left[\frac{\langle\tau \mid s\rangle}{\sqrt{\langle\tau \mid \tau\rangle\langle s \mid s\rangle}}\right]_{\left\{\vec{\theta}_{\tau}=\vec{\theta}_{s}, \phi_{0 s}=0\right\}} .
$$

We find it convenient to discuss results also in terms of the unfaithfulness, that is, $\overline{\mathcal{F}}=1-\mathcal{F}$. Henceforth, we consider the NR waveform as the signal and the SEOBNR_NSBH or PhenomNSBH as the template. In Table III we list the

TABLE III. We list the unfaithfulness between different waveform models and SXS NSBH simulations. To compute the unfaithfulness we use the Advanced LIGO design sensitivity PSD. In the left columns we show the unfaithfulness between SEOBNR_NSBH and PhenomNSBH, and the NR simulations. We compute the mismatch integral starting at the lower frequency $f_{\text {low }}$ listed; this corresponds to a time late enough in the waveform that effects of junk radiation and eccentricity are negligible. The simulations below the horizontal line indicate simulations with nonzero $\chi_{\text {NS }}$. These simulations were not included in the calibration and are used for validation.

\begin{tabular}{lccc}
\hline \hline Simulation & $f_{\text {low }}[\mathrm{Hz}]$ & SEOBNR_NSBH PhenomNSBH \\
\hline SXS:BHNS:0001 & 169 & $7.5 \times 10^{-3}$ & $8.8 \times 10^{-3}$ \\
SXS:BHNS:0002 & 315 & $6.0 \times 10^{-3}$ & $4.5 \times 10^{-3}$ \\
SXS:BHNS:0003 & 407 & $5.5 \times 10^{-3}$ & $5.2 \times 10^{-3}$ \\
SXS:BHNS:0004 & 447 & $7.8 \times 10^{-3}$ & $1.9 \times 10^{-2}$ \\
SXS:BHNS:0006 & 314 & $5.6 \times 10^{-3}$ & $5.3 \times 10^{-3}$ \\
M12-7-S8-LS220 & 351 & $6.9 \times 10^{-3}$ & $7.8 \times 10^{-3}$ \\
M12-7-S9-LS220 & 343 & $1.1 \times 10^{-2}$ & $7.4 \times 10^{-3}$ \\
M12-10-S8-LS220 & 279 & $8.4 \times 10^{-3}$ & $2.0 \times 10^{-2}$ \\
M12-10-S9-LS220 & 271 & $1.1 \times 10^{-2}$ & $2.7 \times 10^{-2}$ \\
M14-7-S7-LS220 & 431 & $1.6 \times 10^{-2}$ & $9.3 \times 10^{-3}$ \\
M14-7-S8-LS220 & 397 & $1.1 \times 10^{-2}$ & $2.0 \times 10^{-2}$ \\
M14-7-S9-LS220 & 426 & $1.7 \times 10^{-2}$ & $1.4 \times 10^{-2}$ \\
M14-10-S8-LS220 & 286 & $1.1 \times 10^{-2}$ & $4.6 \times 10^{-2}$ \\
M14-10-S9-LS220 & 297 & $1.1 \times 10^{-2}$ & $4.3 \times 10^{-2}$ \\
\hline SXS:BHNS:0005 & 448 & $7.4 \times 10^{-2}$ & $8.0 \times 10^{-2}$ \\
SXS:BHNS:0007 & 315 & $7.3 \times 10^{-3}$ & $1.1 \times 10^{-2}$ \\
Q3S9 & 300 & $8.2 \times 10^{-3}$ & $4.9 \times 10^{-3}$ \\
Q4S9 & 238 & $1.0 \times 10^{-2}$ & $6.5 \times 10^{-3}$ \\
\hline \hline
\end{tabular}


unfaithfulness obtained against all the SXS NSBH waveforms at our disposal, for both SEOBNR_NSBH and PhenomNSBH. We also specify the lower frequency $f_{\text {low }}$ used to compute the match. We see that both models have broadly similar performance.

Since the NR waveforms do not cover the entire bandwidth of the detector, we compute the faithfulness also between both NSBH waveform models and NR hybrids. We construct hybrids with both SEOBNR_NSBH and PhenomNSBH and compare both waveform models to the two hybrids. The four comparisons have two distinct purposes. First, the low-frequency part of SEOBNR_NSBH and the SEOBNR_NSBH hybrid, and the PhenomNSBH and PhenomNSBH hybrid, are identical up to a shift in the time and phase of the waveform, so that the unfaithfulness of SEOBNR_NSBH with an SEOBNR_NSBH hybrid quantifies the error of the waveform model failing to capture the NR; the same is true of the unfaithfulness between PhenomNSBH and a PhenomNSBH hybrid. Second, comparing SEOBNR_NSBH with a PhenomNSBH hybrid, and vice versa, includes the error from the NR part of the waveform, and additionally the error of waveform modeling uncertainty. We show the results in Table IV. We note that the choice of hybrid affects the unfaithfulness: the unfaithfulness of SEOBNR_NSBH with PhenomNSBH hybrids tends to be larger than the unfaithfulness of PhenomNSBH with SEOBNR_NSBH hybrids.

To construct the hybrids, we follow the hybridization procedure given in Refs. [59,88]. We first align the waveforms by adjusting the time and phase of SEOBNR_NSBH to maximize the overlap with the NR waveform, and then we apply a Hann window to smoothly transition from the model to the NR waveform. We refer to the initial and final times of the alignment window as $t_{\text {min }}$ and $t_{\max }$, respectively. These are chosen for each waveform to produce good agreement in the early part of the waveform. We provide the windows used in Table IV.

In Fig. 4, we compare the frequency-domain amplitude of the SEOBNR_NSBH model and PhenomNSBH against two publicly available nonspinning SXS waveforms which were used to calibrate SEOBNR_NSBH. For context, we additionally show the BBH baseline model, SEOBNR_BBH. For SXS:BHNS:0001, which is a nondisruptive merger, the amplitudes of the NR data, SEOBNR_BBH, SEOBNR_NSBH, and PhenomNSBH, agree well. For the disruptive merger SXS:BHNS:0002, SEOBNR_NSBH and PhenomNSBH capture the tapering of the amplitude due to tidal disruption. In Fig. 5, we compare SEOBNR_NSBH and PhenomNSBH to the same two NR simulations in the time domain. We include the NR error for those waveforms for which it is available, estimated using the methods described in Refs. [73,94]. In Fig. 6, we compare SEOBNR_NSBH and PhenomNSBH to the accurate spinning simulations Q3S9 and Q4S9, which we use for validation. We align the waveforms using the same procedure to construct the hybrids. We perform these comparisons using the $N=3$ extrapolation order.

TABLE IV. Unfaithfulness between the PhenomNSBH and SEOBNR_NSBH waveform models, and NR hybrids constructed with these models. The details of the hybrid construction are given in the main text. We give here the initial $t_{\min }$ and final $t_{\max }$ times of the window used for hybridization in seconds, relative to the beginning of the NR data.

\begin{tabular}{|c|c|c|c|c|c|c|}
\hline \multirow[b]{2}{*}{ Simulation } & \multicolumn{2}{|c|}{ Window } & \multicolumn{2}{|c|}{ SEOBNR_NSBH hybrid } & \multicolumn{2}{|c|}{ PhenomNSBH hybrid } \\
\hline & $t_{\min }[\mathrm{s}]$ & $t_{\max }[\mathrm{s}]$ & SEOBNR_NSBH & PhenomNSBH & SEOBNR_NSBH & PhenomNSBH \\
\hline SXS:BHNS:0001 & 0.01 & 0.025 & $1.2 \times 10^{-3}$ & $1.2 \times 10^{-2}$ & $2.4 \times 10^{-3}$ & $1.3 \times 10^{-2}$ \\
\hline SXS:BHNS:0002 & 0.01 & 0.025 & $7.4 \times 10^{-4}$ & $1.3 \times 10^{-4}$ & $1.3 \times 10^{-3}$ & $1.4 \times 10^{-4}$ \\
\hline SXS:BHNS:0003 & 0.005 & 0.018 & $2.1 \times 10^{-4}$ & $1.6 \times 10^{-4}$ & $2.7 \times 10^{-3}$ & $1.3 \times 10^{-3}$ \\
\hline SXS:BHNS:0004 & 0.008 & 0.02 & $1.6 \times 10^{-4}$ & $5.7 \times 10^{-4}$ & $9.1 \times 10^{-4}$ & $2.7 \times 10^{-4}$ \\
\hline SXS:BHNS:0006 & 0.01 & 0.025 & $1.8 \times 10^{-3}$ & $1.9 \times 10^{-3}$ & $4.6 \times 10^{-3}$ & $3.9 \times 10^{-4}$ \\
\hline M12-7-S8-LS220 & 0.008 & 0.026 & $2.0 \times 10^{-3}$ & $1.8 \times 10^{-2}$ & $1.8 \times 10^{-2}$ & $4.2 \times 10^{-3}$ \\
\hline M12-7-S9-LS220 & 0.01 & 0.03 & $1.4 \times 10^{-3}$ & $5.0 \times 10^{-3}$ & $1.8 \times 10^{-2}$ & $3.2 \times 10^{-3}$ \\
\hline M12-10-S8-LS220 & 0.01 & 0.04 & $7.1 \times 10^{-3}$ & $1.9 \times 10^{-2}$ & $6.2 \times 10^{-2}$ & $1.4 \times 10^{-2}$ \\
\hline M12-10-S9-LS220 & 0.01 & 0.04 & $1.1 \times 10^{-2}$ & $2.9 \times 10^{-2}$ & $7.2 \times 10^{-2}$ & $2.5 \times 10^{-2}$ \\
\hline M14-7-S7-LS220 & 0.0075 & 0.02 & $9.1 \times 10^{-4}$ & $5.3 \times 10^{-3}$ & $5.5 \times 10^{-3}$ & $2.9 \times 10^{-4}$ \\
\hline M14-7-S8-LS220 & 0.006 & 0.018 & $3.7 \times 10^{-4}$ & $9.1 \times 10^{-3}$ & $2.6 \times 10^{-3}$ & $2.2 \times 10^{-3}$ \\
\hline M14-7-S9-LS220 & 0.0075 & 0.02 & $1.6 \times 10^{-3}$ & $3.7 \times 10^{-3}$ & $7.1 \times 10^{-3}$ & $3.3 \times 10^{-4}$ \\
\hline M14-10-S8-LS220 & 0.005 & 0.03 & $1.4 \times 10^{-3}$ & $2.4 \times 10^{-2}$ & $3.6 \times 10^{-2}$ & $4.3 \times 10^{-3}$ \\
\hline M14-10-S9-LS220 & 0.007 & 0.03 & $1.1 \times 10^{-2}$ & $6.5 \times 10^{-2}$ & $3.3 \times 10^{-2}$ & $4.3 \times 10^{-3}$ \\
\hline SXS:BHNS:0005 & 0.005 & 0.015 & $3.4 \times 10^{-3}$ & $4.0 \times 10^{-2}$ & $4.7 \times 10^{-2}$ & $3.6 \times 10^{-3}$ \\
\hline SXS:BHNS:0007 & 0.005 & 0.055 & $6.4 \times 10^{-4}$ & $1.9 \times 10^{-2}$ & $7.1 \times 10^{-3}$ & $1.2 \times 10^{-3}$ \\
\hline Q3S9 & 0.01 & 0.025 & $5.5 \times 10^{-4}$ & $1.2 \times 10^{-2}$ & $1.5 \times 10^{-2}$ & $3.3 \times 10^{-4}$ \\
\hline Q4S9 & 0.01 & 0.025 & $8.6 \times 10^{-4}$ & $2.4 \times 10^{-3}$ & $6.9 \times 10^{-3}$ & $2.4 \times 10^{-3}$ \\
\hline
\end{tabular}



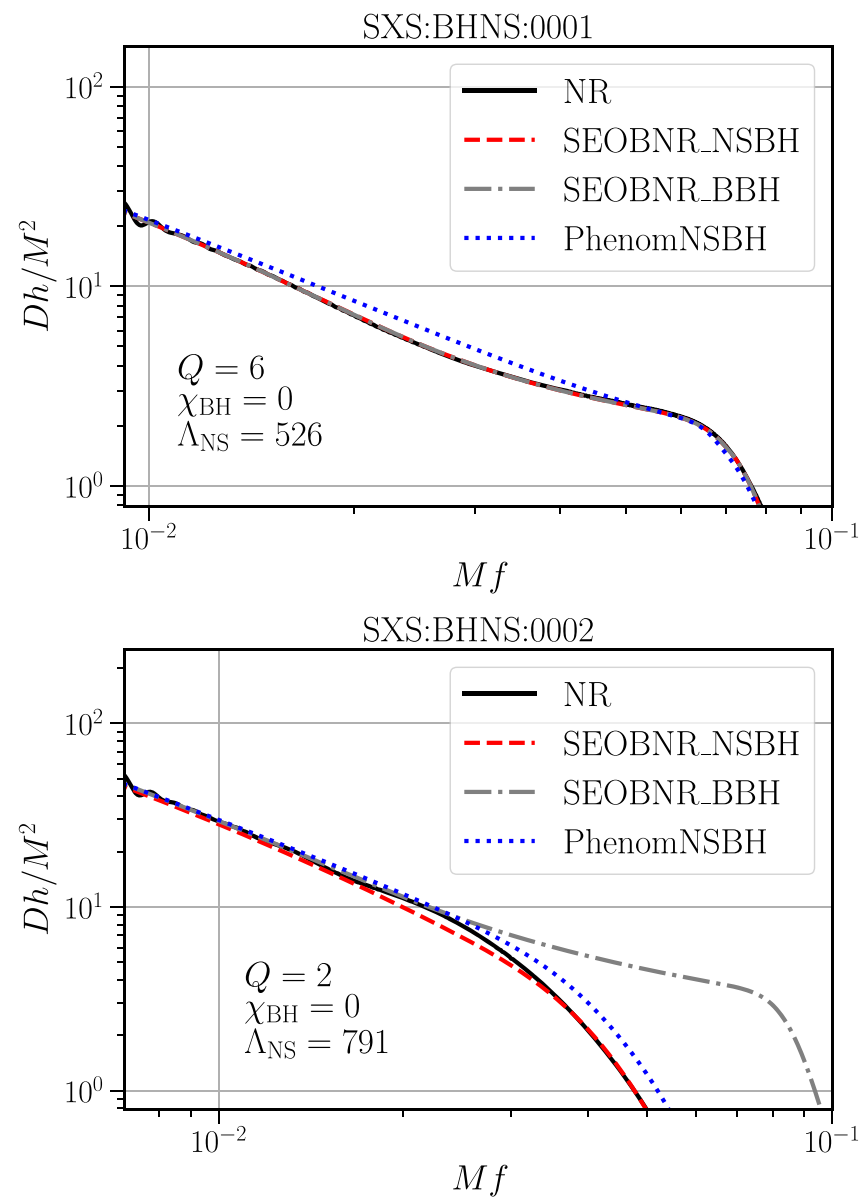

FIG. 4. Frequency-domain amplitude comparisons of SXS simulations, the NSBH waveform models SEOBNR_NSBH and PhenomNSBH, and the BBH model SEOBNR_BBH, that is used as a baseline for SEOBNR_NSBH. SEOBNR_NSBH is able to capture the effects of tidal disruption on the amplitude, while also reducing to $\mathrm{BBH}$-like waveform for large mass ratios when tidal disruption does not occur.

\section{E. Regime of validity}

In Table V, we provide the parameter space region of the simulations used for calibration. We also give a suggested regime of validity for use of our SEOBNR_NSBH waveform model, which we justify as follows.

(i) Mass ratio Q.-We take the lower limit for the mass ratio to be 1 , given that in our fit we include NR simulations with these mass ratios. For large enough mass ratios, for any spin and $\Lambda_{\mathrm{NS}}$, the merger becomes nondisruptive and the model reduces to the SEOBNR_BBH waveform model. We have checked that there is always a range of parameter space at large mass ratios where this transition occurs, within the regime of validity of the model. Therefore we inherit the upper limit on $Q$ coming from SEOBNR_BBH, which is of 100.

(ii) NS mass $M_{\mathrm{NS}}$.-Based on expectations of the maximum NS mass from the nuclear EOS, we

restrict the NS mass to be less than $3 M_{\odot}$. We also suggest restricting the NS mass to be larger than $1 M_{\odot}$, which is consistent with the range that we choose for the tidal parameter $\Lambda_{\mathrm{NS}}$.

(iii) NS tidal-deformability $\Lambda_{\mathrm{NS}}$. - We have verified that sensible waveforms are generated with $\Lambda_{\mathrm{NS}}$ varying from 0 up to 5000 and on this basis suggest the waveform model can be used in this range. We have also performed a calibration and comparison against available NR simulations to verify the model accurately describes simulations with tidal disruption, as we have described. However the available NR simulations have a more limited range of $\Lambda_{\mathrm{NS}}$, depending on the NS mass and equation of state, as seen in Fig. 2. Thus we caution that tidal-disruption effects are uncertain for large $\Lambda_{\mathrm{NS}}$, in particular $\Lambda_{\mathrm{NS}} \gtrsim 1000$ for $1.4 M_{\odot}$ NSs. Even this restricted range includes the bound $\Lambda_{\mathrm{NS}}<800$ for a $1.4 M_{\odot} \mathrm{NS}$, obtained from measurements of GW170817 in Ref. [95].

(iv) $B H \operatorname{spin} \chi_{\mathrm{BH}}$.- - In the fit we include simulations with positive spins as large as 0.9 and negative spins as low as -0.5 . Since negative spins tend to make the merger less disruptive (i.e., more BBH-like), in order to obtain a symmetric range we suggest $[-0.9,0.9]$ as a range for the spin.

(v) NS spin $\chi_{\mathrm{NS}}$.-While we do not include simulations with NS spin in the fit, from PN theory we expect that the main effect of the spin enters via the beta parameter derived in Ref. [96]; here we use the formulation given in Eq. (A6) of Ref. [97]:

$$
\beta=\frac{113-76 \eta}{12} \chi_{\mathrm{eff}}+\frac{76 \delta}{12} \chi_{a}
$$

where $\eta=Q /(Q+1)^{2}$ is the symmetric mass ratio, $\chi_{a}=\left(\chi_{\mathrm{BH}}-\chi_{\mathrm{NS}}\right) / 2$ is the antisymmetric combination of aligned spins, $\delta=(Q-1) /(Q+1)$ is an antisymmetric combination of the masses, and the effective aligned-spin parameter $\chi_{\text {eff }}$ is given by

$$
\chi_{\mathrm{eff}}=\frac{M_{\mathrm{BH}} \chi_{\mathrm{BH}}+M_{\mathrm{NS}} \chi_{\mathrm{NS}}}{M_{\mathrm{BH}}+M_{\mathrm{NS}}} .
$$

Except for mass ratios close to 1 and small spins, $\beta$ is dominated by the $\mathrm{BH}$ spin. We also see reasonable agreement with simulations when the NS spin is nonzero, as shown in Table III. We therefore recommend that the NS spin is bounded by the low-spin prior that has been used in the literature (e.g., Refs. [3,4]), $\left|\chi_{\mathrm{NS}}\right|<0.05$.

Through a thorough study, we have verified that the SEOBNR_NSBH waveforms look sensible in the region in which we suggest to use this model. 

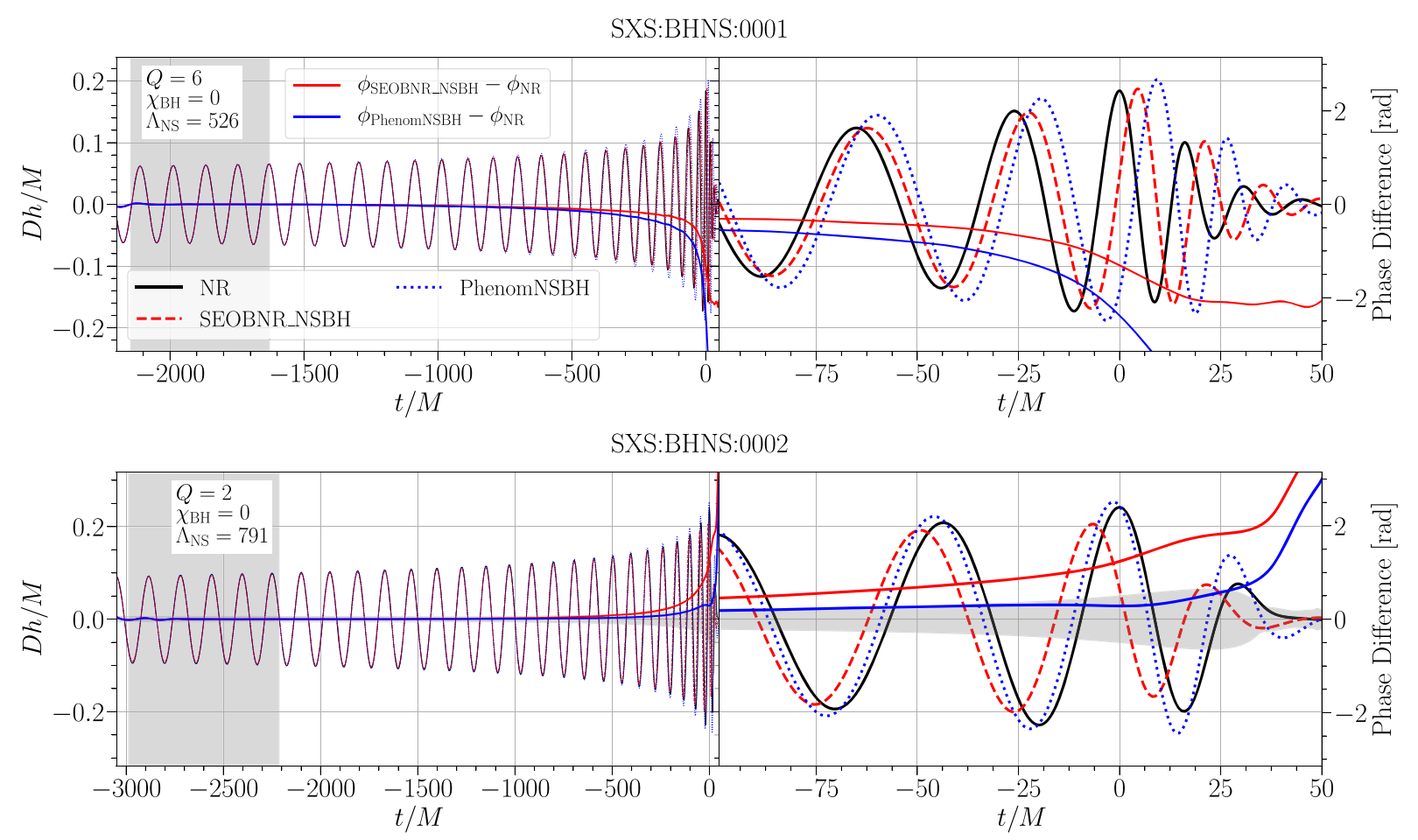

FIG. 5. Time domain comparisons of two NR simulations in the time domain, along with SEOBNR_NSBH and PhenomNSBH. We show two of the publicly available SXS simulations with zero spin which were used for calibration of SEOBNR_NSBH: the nondisruptive merger SXS:BHNS:0001, with mass ratio 6, and the disruptive merger SXS:BHNS:0002 with mass ratio 2. Also plotted is the phase difference for both NSBH models against the relevant NR simulation. The gray band shows the region used to align the model waveforms and NR. We also show the NR phase error for SXS:BHNS:0002 as a horizontal gray band; for SXS:BHNS:0001, which is an older waveform, the NR error is not available. The NR waveform has been shifted in time so the peak amplitude occurs at $t=0$ and that the phase is zero there. We see that, for both waveform families, the agreement with NR is very good at the beginning of the NR waveform, but there is dephasing toward the end.

\section{APPLICATIONS}

Having constructed the SEOBNR_NSBH waveform model and checked that it agrees well with existing NR waveforms, we now apply the model to three data-analysis problems. In particular, in Sec. III A, we compute the unfaithfulness of the SEOBNR_NSBH model against SEOBNR_BBH and SEOBNR_BNS models in order to obtain an estimate of the regions of parameter space where the advanced-detector network may be able to distinguish different source classes. In Sec. III B, we perform a Bayesian parameter-estimation analysis in which we inject a synthetic NSBH signal (notably a disruptive NSBH merger) and infer the source's properties and parameter's biases when recovering it with the SEOBNR_NSBH model and the SEOBNR_BBH model. Finally, in Sec. III C, we reanalyze the LIGO/Virgo event GW170817 under the hypothesis that it is a NSBH binary, instead of a BNS.

\section{A. Distinguishing different source classes}

When is it possible to determine whether a given binary system is a BBH, BNS, or NSBH based on tidal effects in the gravitational waveform? We can address this question with our waveform model by considering how similar a SEOBNR_NSBH waveform is to a waveform from another source class. In this section we do not use an astrophysical prior on the masses of the objects to distinguish the source classes. Reference [98] considered the issue of distinguishing source classes, using measurements on the masses of the component objects, and an astrophysical prior on the masses of NSs and BHs, rather than measurements of the tidal parameter which we consider here. The conclusion of that work is that it will be difficult to distinguish different source classes with Advanced LIGO, with signals with signal-to-noise ratios (SNRs) in the range 10-20.

First, we consider the case of distinguishing the hypotheses that a given signal is a BBH or a NSBH. Suppose the signal is a NSBH with a given set of parameters, $\vec{\theta}_{\mathrm{NSBH}}$. We compute the unfaithfulness between the SEOBNR_NSBH and SEOBNR_BBH models, with the same masses and spins. In the left panel of Fig. 7, we show contours of the unfaithfulness in the $Q-\Lambda_{\mathrm{NS}}$ plane, for a $1.4 M_{\odot} \mathrm{NS}$ and $\chi_{\mathrm{NS}}=0$, while varying $\chi_{\mathrm{BH}}$ over the range $\{0,0.5,0.9\}$. To put the results in context, following Ref. [99], we estimate that two waveforms are distinguishable at the $1 \sigma$ level when the SNR $\rho$ satisfies $\overline{\mathcal{F}}=K / 2 \rho^{2}$, where $K$ depends on the 

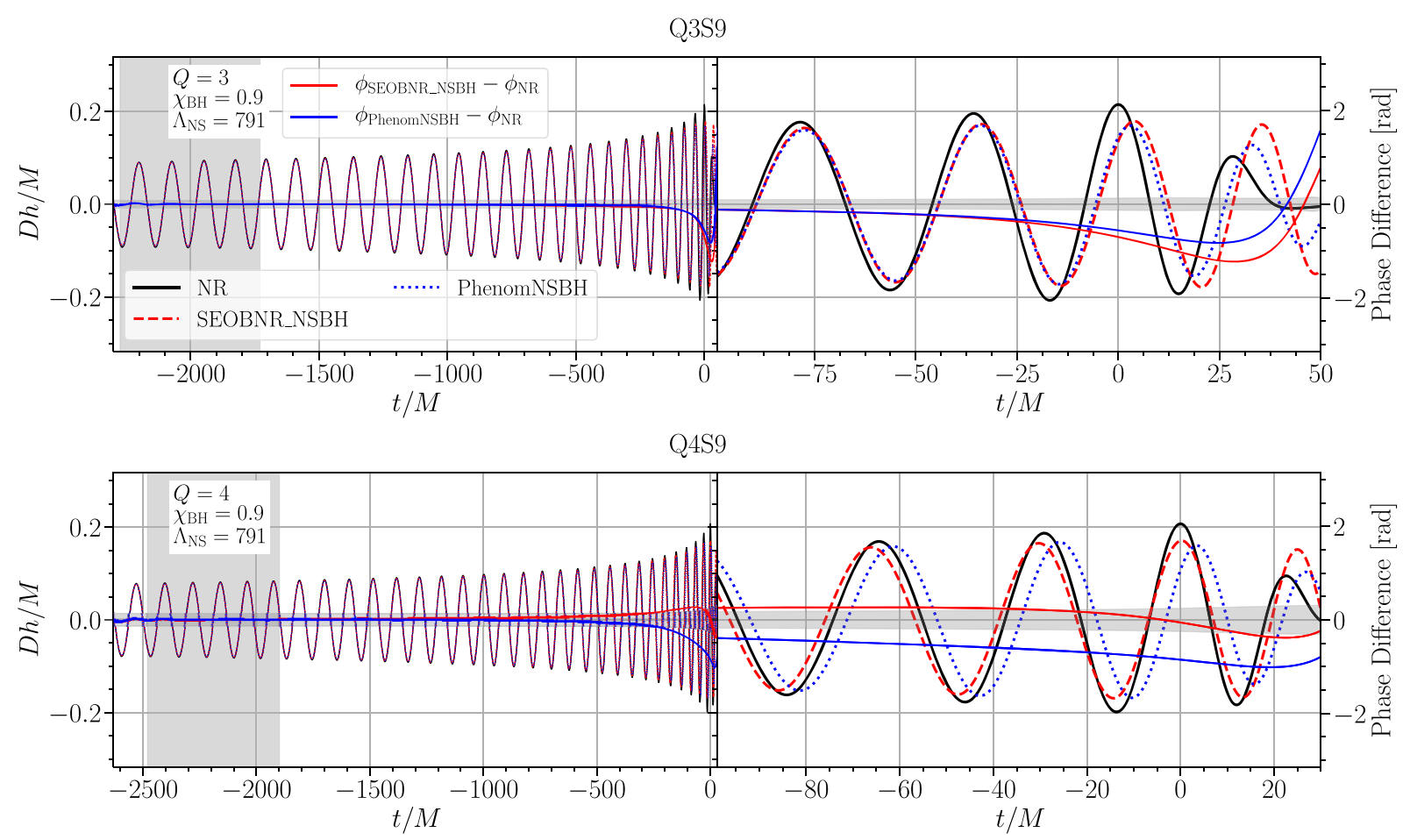

FIG. 6. Time domain comparisons of highly accurate waveforms of disruptive mergers, as well as the models SEOBNR_NSBH and PhenomNSBH. We show Q3S9, with mass ratio 3, and Q4S9, with mass ratio 4. Both configurations have a dimensionless $\mathrm{BH}$ spin magnitude of 0.9. These waveforms were not used to calibrate SEOBNR_NSBH. We see excellent agreement across a large number of cycles. The time and phase of the NR waveform have been fixed as in Fig. 5.

number of intrinsic parameters, D. Reference [100] provides an estimate of $K=D-1$, at which the $D$ dimensional posteriors do not overlap at the $1 \sigma$ level. Then, an unfaithfulness of $\overline{\mathcal{F}}=10^{-3}$ corresponds to an SNR of $\rho \approx 45$. However, this criterion does not apply directly to marginalized posteriors. A more detailed discussion of the use of this criterion can be found in Ref. [101]. In particular, the value of $K$ at which systematic errors become comparable to statistical ones depends on

TABLE V. Range of intrinsic parameters for which the SEOBNR_NSBH waveform model was calibrated and in which we suggest the model can be used. The calibration range gives the region of parameter space for which there are NR simulations. The suggested range of validity is the range we suggest for using the waveform, as explained in the main text. We note that a real EOS relates $\Lambda_{\mathrm{NS}}$ and $M_{\mathrm{NS}}$, and $\Lambda_{\mathrm{NS}}$ is a rapidly decreasing function of $M_{\mathrm{NS}}$. The largest value of $\Lambda_{\mathrm{NS}}$ for a $1.4 M_{\odot} \mathrm{NS}$ among the simulations we use is 791 . See the main text for more detailed discussion.

\begin{tabular}{lcc}
\hline \hline Parameter & Calibration range & $\begin{array}{c}\text { Suggested range } \\
\text { of validity }\end{array}$ \\
\hline$Q$ & {$[1,6]$} & {$[1,100]$} \\
$M_{\mathrm{NS}}$ & {$[1.2,1.4] M_{\odot}$} & {$[1,3] M_{\odot}$} \\
$\Lambda_{\mathrm{NS}}$ & {$[130,4200]$} & {$[0,5000]$} \\
$\chi_{\mathrm{BH}}$ & {$[-0.5,0.9]$} & {$[-0.9,0.9]$} \\
\hline \hline
\end{tabular}

what parameter is being considered, as well as extrinsic parameters such as the inclination. They find that, when applying the criterion to marginalized posteriors, the estimate $K=D-1$ is conservative. Therefore, we

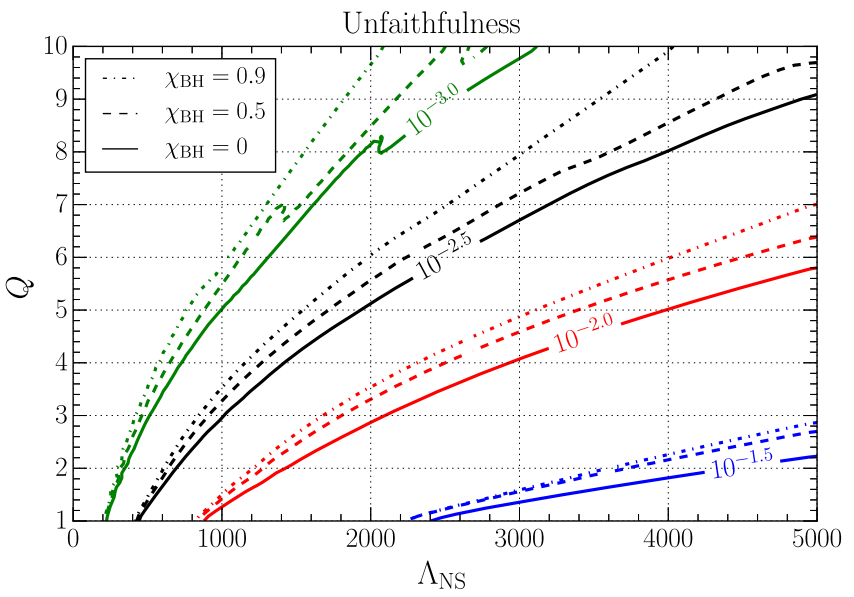

FIG. 7. Contours with constant unfaithfulness in the $Q-\Lambda_{\mathrm{NS}}$ plane for varying $\mathrm{BH}$ spin $\chi_{\mathrm{BH}}$, when comparing SEOBNR_BBH and SEOBNR_NSBH assuming the Advanced LIGO design sensitivity PSD. As discussed in the main text, the unfaithfulness can be used to provide an estimate of the SNR at which data can be used to distinguish between two waveforms. Note that it is easier to distinguish $\mathrm{BBH}$ and $\mathrm{NSBH}$ systems for smaller $Q$ and larger $\chi_{\mathrm{BH}}$. 
emphasize that this criterion is sufficient, but it is not necessary, and also it does not say which parameters are biased and by how much.

We also compute the unfaithfulness between the SEOBNR_NSBH and SEOBNR_BNS models, with the same masses, spins, and tidal parameters. We find that, for zero spin, the unfaithfulness between NSBH and BNS is always less than $10^{-3}$ when the NS mass is less than $3 M_{\odot}$. This suggests it will be very difficult to distinguish NSBH and BNS systems on the basis of tidal effects on the waveform alone. However, inference on the component masses provides additional useful information that can help distinguish different source classes.

As said above, computing the unfaithfulness does not allow us to quantify its impact on the inference of the parameters of the binary and quantify possible biases. Therefore, in the next section, at least for one particular case, we perform a Bayesian parameter-estimation study, extract those biases, and compare with the distinguishability criterion of Refs. [99,100].

\section{B. Parameter-estimation case study}

In this section, because of computational costs, we perform a Bayesian parameter-estimation analysis for one specific NSBH system and postpone to the future a more comprehensive analysis.

We first create a synthetic NSBH signal consisting of an NR hybrid built by stitching together the SEOBNR_NSBH waveform to the SXS:BHNS:0006 waveform, with masses $M_{\mathrm{BH}}=2.1 M_{\odot}, M_{\mathrm{NS}}=1.4 M_{\odot}$, mass ratio $Q=1.5$, and both spins equal to zero. We do not add a noise realization (i.e., we work in zero noise) which is equivalent to averaging over different noise realizations, as shown in Ref. [102]. We perform four injections, with SNRs of 25, 50, 75, and 100 in the Advanced LIGO-Virgo network. While the masses are not astrophysically motivated, this system is interesting to study because it is disruptive, and due to the mass ratio the tidal dephasing is enhanced. Further, SXS:BHNS:0006 is the simulation with the largest number of cycles of the publicly available SXS waveforms.

We apply the Markov chain Monte Carlo (MCMC) sampling algorithm implemented in LALInference [103] to these four signals and recover the signal with both the SEOBNR_BBH and SEOBNR_NSBH waveform models. Due to limited computational resources, we run the parameter estimation with a lower cutoff frequency of $30 \mathrm{~Hz}$. We take the higher cutoff frequency to be $2048 \mathrm{~Hz}$. We use a uniform prior on the detector frame component masses. For SEOBNR_NSBH, we impose a constraint that $M_{\mathrm{NS}}<$ $3 M_{\odot},\left|\chi_{\mathrm{NS}}\right|<0.05$, and $\left|\chi_{\mathrm{BH}}\right|<0.9$ consistent with the range of validity of the model. We take a prior on $\Lambda_{\mathrm{NS}}$ that is uniform between 0 and 5000. For SEOBNR_BBH, we do not impose a constraint on the maximum mass but do require that the spins of both objects were less than 0.9. Since the two approximants make different assumptions about the nature of the component objects, in describing the results of the Bayesian analysis, we refer to the masses as $m_{1}$ and $m_{2}$ rather than $M_{\mathrm{BH}}$ and $M_{\mathrm{NS}}$.

In Fig. 8, we show posteriors in the $m_{1}-m_{2}$ plane, as well as the $q-\chi_{\text {eff }}$ plane, for the $\mathrm{SNR}=25$ and $\mathrm{SNR}=75$ injections. For ease of comparison with other parameterestimation (PE) results by LIGO-Virgo analyses, we show the posterior in terms of the mass ratio $q \equiv Q^{-1}=m_{2} / m_{1}$. For the SNR $=25$ injection, we see the posteriors from the two waveforms agree very well and are consistent with the injected value within the $90 \%$ credible interval. For larger SNRs, posteriors derived using the two waveforms are in tension, and at large enough SNR, the injected value lies outside of the $90 \%$ credible interval of the posterior for each model. For the SNR $=50$ injection, and for larger SNRs, there is a bias in the masses and $\chi_{\text {eff }}$ recovered using SEOBNR_BBH. In particular, SEOBNR_BBH recovers a larger total mass. The biases in the mass are due to the lack of tidal effects in SEOBNR_BBH. To quantify this, we have performed a run with two modified versions of SEOBNR_NSBH. The first modified model has a tidalphase correction but the same amplitude as SEOBNR_BBH. The second one has the tidal-disruption correction to the amplitude but no tidal phase is applied. At SNR $=25$, both models recover the injected mass ratio inside of the $90 \%$ credible interval. At $\mathrm{SNR}=75$, we find that the first modified model, like SEOBNR_NSBH, obtains the correct value within the $90 \%$ interval. On the other hand, the second model, with only the amplitude correction, does not. This is consistent with the fact that the tidal phase accumulates over many cycles, while the merger frequency is at relatively high frequencies outside of the most sensitive band of the detector. ${ }^{2}$ The masses and spins recovered by SEOBNR_NSBH are consistent at the $90 \%$ level with the injected values for the SNR $=50$ case but are only marginally consistent for the $\mathrm{SNR}=75$ injection, and for $\mathrm{SNR}=100$, the injected values of the masses and $\chi_{\text {eff }}$ lie outside $90 \%$ credible interval. This bias is due to differences with the NR-hybrid waveform. We show the recovery of the SNR $=75$ injection, for which the SEOBNR_NSBH recovery is marginally consistent with the true parameters, in the right two panels of Fig. 8.

In Fig. 9, we show recovery of the tidal parameter $\Lambda_{\mathrm{NS}}$ obtained using SEOBNR_NSBH for the four different cases. In all four scenarios, the injected tidal parameter is consistent with the $90 \%$ credible interval of the $\Lambda_{\mathrm{NS}}$ posterior, although this is only marginally true for the $\mathrm{SNR}=100$ injection. It is interesting to compare the difference between the recovered and injected values with what is expected from the indistinguishability criterion discussed in the previous section. The unfaithfulness from $30 \mathrm{~Hz}$ between the NR hybrid used and SEOBNR_NSBH

\footnotetext{
${ }^{2}$ We thank the anonymous referee for suggesting that the bias in this case can be attributable to the tidal phase.
} 

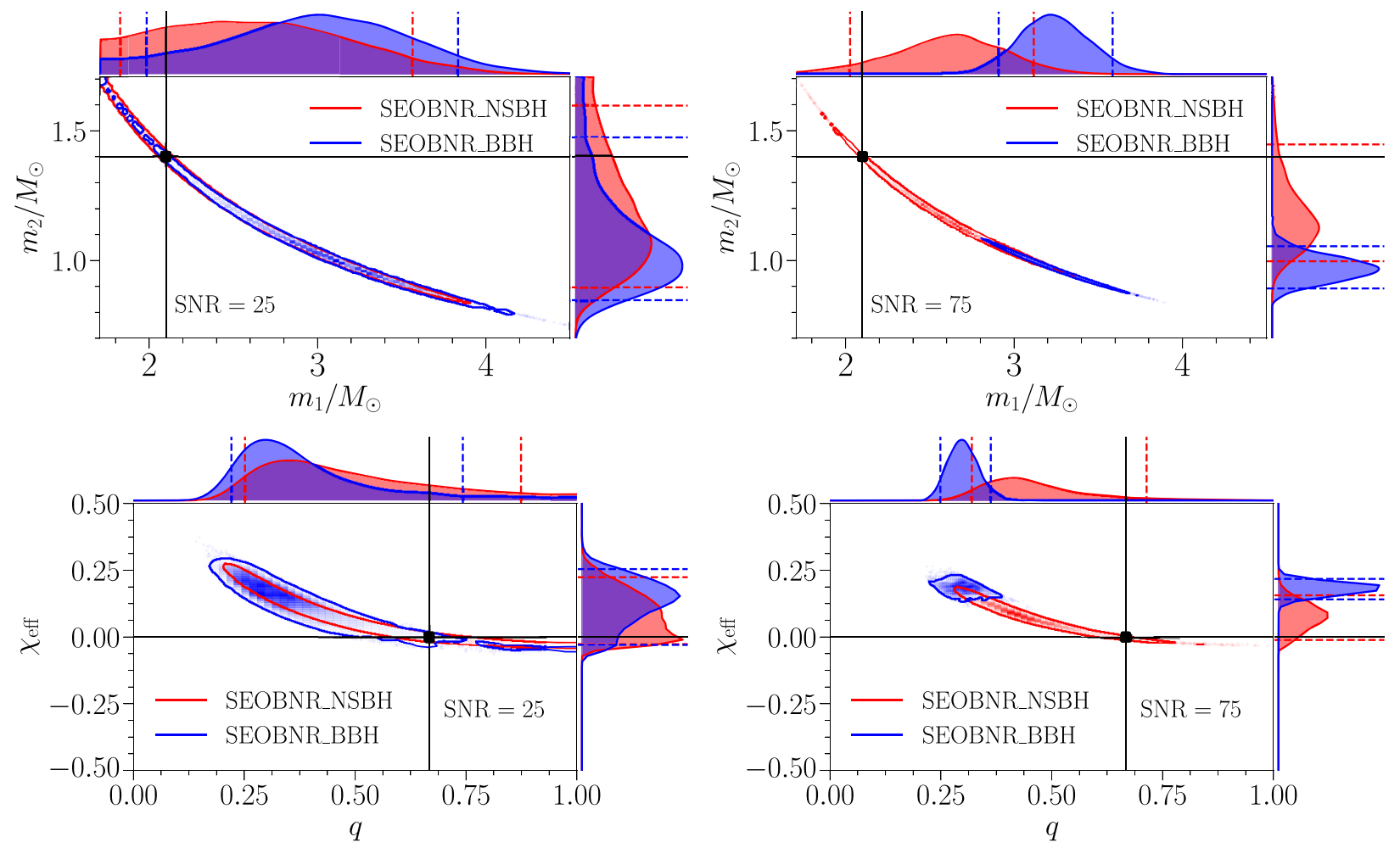

FIG. 8. Illustrative parameter-estimation results for the SXS:BHNS:0006 hybrid injections described in the main text. In the top two panels, we show posterior distributions and $90 \%$ credible intervals for the component masses, with the posteriors derived using the SEOBNR_NSBH (SEOBNR_BBH) approximant in red (blue). In the bottom panels, we show the $q-\chi$ eff plane. We show the injected value as a black dot. For SNR $=25$, both SEOBNR_NSBH and SEOBNR_BBH recover the injected value within the 90\% credible interval. For larger SNRs, the recovery with SEOBNR_BBH is biased. The posterior with the BBH waveform is peaked around a larger total mass than the injected one. We show this explicitly for SNR $=75$. Additionally, we use this software injection test to explore how the difference between SEOBNR_NSBH and the NR hybrid affects parameter estimation. We see that, at SNR = 75, the injected values of the masses and $\chi_{\text {eff }}$ are marginally consistent with the SEOBNR_NSBH posterior at the $90 \%$ level; at larger SNRs we find the $90 \%$ credible interval does not include the true value.

is $2 \times 10^{-3}$, using the Advanced LIGO design sensitivity PSD. From the indistinguishability criterion of Ref. [100] discussed in the previous section, we would expect to see deviations at the $1 \sigma$ level between the posterior recovered with SEOBNR_NSBH and the injected value an SNR of

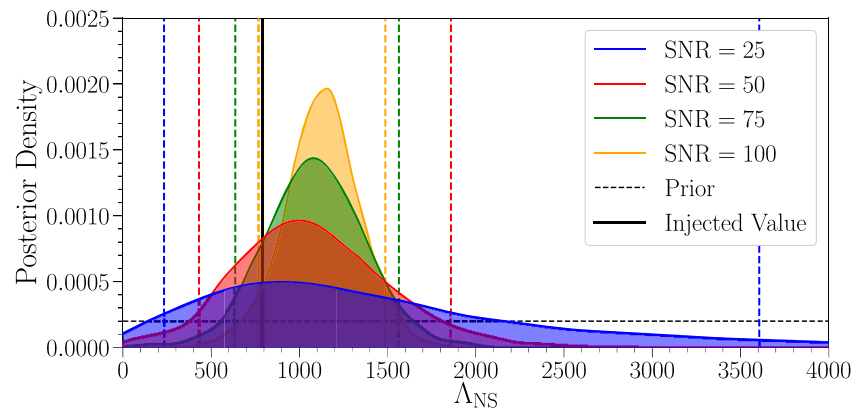

FIG. 9. Posteriors for the NS tidal deformability $\Lambda_{\mathrm{NS}}$ for the NSBH hybrid injections described in the main text, recovered with SEOBNR_NSBH, for different SNRs. The recovered values of $\Lambda_{\mathrm{NS}}$ are consistent with the true value from the hybrid at the $90 \%$ level; however, this is only marginally true for SNR $=100$.
32. A full Bayesian analysis reveals that this level of bias for the recovery of $\tilde{\Lambda}$ only arises at a larger value of the SNR. However as we have emphasized, the criterion strictly applies only to the full $D$-dimensional posterior and not the marginalized posteriors we consider in this section. Additionally, the criterion is only sufficient; it does not specify which parameters are biased, depends on extrinsic parameters such as the inclination, and has been shown to be quite conservative when applied to the marginalized posteriors [101].

This case study illustrates the importance of having accurate NSBH models that can account for tidal disruption in order to derive correct conclusions about astrophysical parameters. However, we emphasize that these injections are only meant as an example. Larger mass ratios may be less tidally disruptive and have tidal effects on the phase suppressed. Conversely, systems with large BH spin will tend to be more disruptive, which will enhance the differences between the BBH and NSBH waveforms.

After this manuscript was submitted, Ref. [104] appeared as a preprint. This work provides a detailed parameter 
estimation study, recovering many different NSBH injections using SEOBNR_NSBH, PhenomNSBH, SEOBNR_BBH, SEOBNR_BNS, and other waveform models. The injected waveforms are hybrids of SXS:BHNS:0001, SXS: BHNS:0003, and SXS:BHNS:0004, with the NR surrogate model NRHybSur3dq8Tidal, developed in Ref. [105], at $\mathrm{SNR}=30$ and SNR $=70$. Of the cases they study, SXS: BHNS:0004, with $M_{\mathrm{NS}}=M_{\mathrm{BH}}=1.4 M_{\odot}$, has the most similar parameters to the signal we consider in this work. The results that Ref. [104] obtains for SXS: BHNS:0004 are broadly similar to the results we present here. When SNR $=30$, the injected masses are recovered within the $90 \%$ credible intervals when recovering with SEOBNR_BBH, SEOBNR_BNS, SEOBNR_NSBH, and PhenomNSBH. When SNR $=70$, the component masses recovered using SEOBNR_BBH are biased toward larger values. On the other hand, the posteriors obtained with SEOBNR_BNS, SEOBNR_NSBH and PhenomNSBH recover the component masses within the $90 \%$ credible interval. The fact that SEOBNR_BNS and SEOBNR_NSBH both recover the correct mass may indicate that the tidal phase is more important than the tidal-disruption frequency for recovering the masses. For the tidal parameter, at $\mathrm{SNR}=70$, the authors find that the recovery of SEOBNR_NSBH as well as SEOBNR_BNS is in tension at the $90 \%$ level. Interestingly, PhenomNSBH recovers the correct tidal parameter when SNR $=70$. This paper, like the current work, illustrates the need for accurate NSBH models as the detector network sensitivity improves.

\section{Inference of GW170817 as a NSBH}

As a final application, we reanalyze GW170817 [106] under the hypothesis that it is a NSBH (see also Refs. [17,18] for related studies). Indeed, it is interesting to ask whether GW data alone can be used to distinguish the hypotheses that this event is a BNS or a NSBH.

We run the Bayesian inference study with the MCMC code implemented in LALInference, using publicly available data of GW170817 from the GW open science center [107] (discussion of these data for $\mathrm{O} 1$ and $\mathrm{O} 2$ is contained in Ref. [108]). We run with both the SEOBNR_NSBH model as well as the SEOBNR_BNS model in order to be able to do a fair comparison. As far as we know, this is the first time that the new version of the SEOBNR_BNS model has been used to analyze GW170817. We compare our results to those from the runs obtained in the GWTC-1 catalog [3], which used a former version of the SEOBNR_BNS model. We use the same priors as the GWTC-1 analysis [3], except where otherwise stated. For SEOBNR_BNS we assume a flat prior on $\Lambda_{1}$ and $\Lambda_{2}$, while for SEOBNR_NSBH we assume a flat prior on $\Lambda_{2}$ and fix $\Lambda_{1}$ to zero. The posteriors contain support only in the interior of the prior domain for both waveform models with these priors. The prior on the component mass ranges from 0.5 to $7.7 M_{\odot}$, and therefore the prior does not require that both objects have masses below the maximum mass of a NS.

First, we obtain that the median-recovered matched-filter SNR for each waveform model is 32.7. Since the SEOBNR_NSBH and SEOBNR_BNS models recover the signal with a similar SNR, we do not find a clear preference either for a NSBH or BNS signal, when we only consider the GW data. Moreover, in Fig. 10, we show the recovery of the mass ratio and tidal deformability $\tilde{\Lambda}$ which is given by

$\tilde{\Lambda}=\frac{16}{13} \frac{\left(m_{1}+12 m_{2}\right) m_{1}^{4} \Lambda_{1}+\left(m_{2}+12 m_{1}\right) m_{2}^{4} \Lambda_{2}}{\left(m_{1}+m_{2}\right)^{5}}$.

In order to more easily compare with results in Ref. [3], we show the mass ratio $q \equiv Q^{-1}=M_{\mathrm{NS}} / M_{\mathrm{BH}}$. There is a preference for unequal mass ratios in the SEOBNR_NSBH case, due to tidal disruption that occurs for higher mass ratios. Since $\tilde{\Lambda}$ depends nontrivially on the mass ratio and
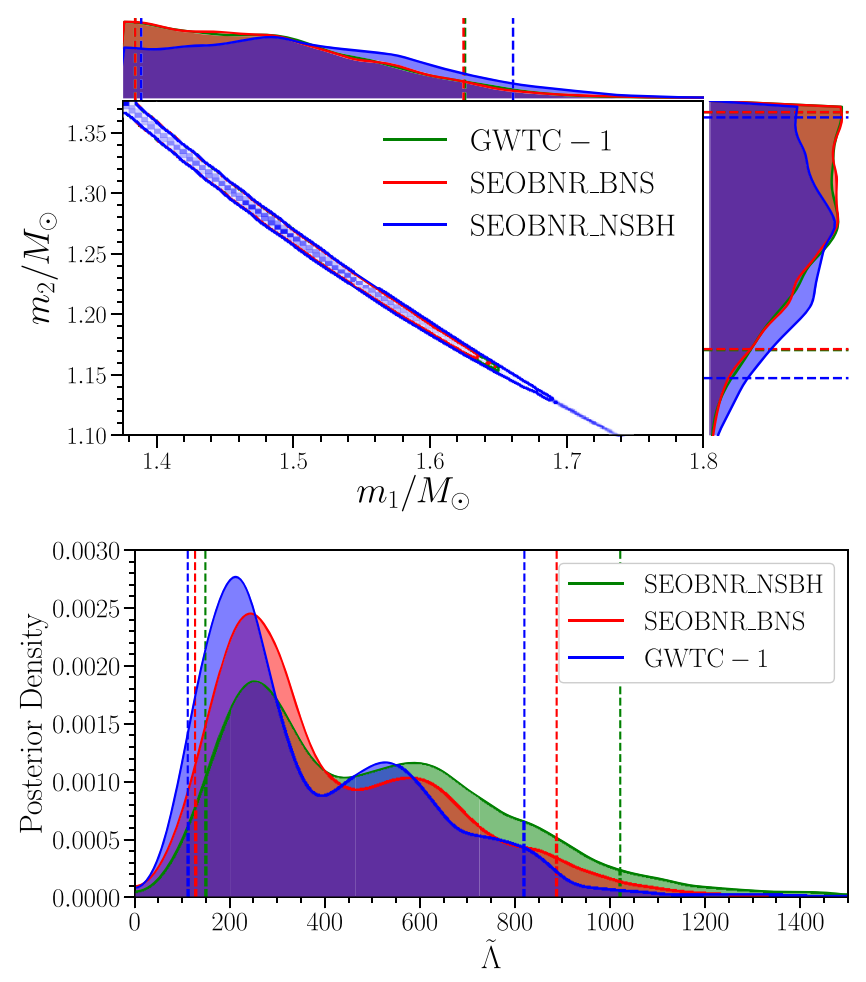

FIG. 10. Reanalysis of GW170817. We show posteriors for the effective tidal deformability $\tilde{\Lambda}$ and the mass ratio $q \equiv m_{2} / m_{1}$, for three waveform models: in green we show the recovery with an older version of SEOBNR_BNS which performed in GWTC-1. In red we show a recovery with the current version of SEOBNR_BNS, which has been recalibrated in Ref. [59]. The results are broadly consistent, though there are small differences consistent with changes to the waveform model. Finally, in blue we show the recovery with SEOBNR_NSBH. The NSBH model has a slight preference for less equal mass ratios. Note that we have reweighted the samples by dividing by the prior on $\tilde{\Lambda}$, as done in Ref. [3]. 
TABLE VI. Median value and 90\% credible region for parameters during a reanalysis of GW170817 with SEOBNR_NSBH and SEOBNR_BNS. The three PE runs give consistent results without a strong preference for BNS or NSBH based on GW data alone. The NSBH recovery appears to have a slight preference for unequal mass ratios and a smaller effective tidal deformability $\tilde{\Lambda}$ compared to the BNS recoveries.

\begin{tabular}{lccccc}
\hline \hline Approximant & $m_{1} / M_{\odot}$ & $m_{2} / M_{\odot}$ & $\chi_{\mathrm{eff}} / 10^{-3}$ & $\tilde{\Lambda}$ & Matched filter SNR \\
\hline SEOBNR_BNS (GWTC-1 version) & $1.47_{-0.09}^{+0.15}$ & $1.28_{-0.11}^{+0.08}$ & $3.4_{-8}^{+14}$ & $456_{-307}^{+565}$ & $32.7_{-0.1}^{+0.1}$ \\
SEOBNR_BNS (current version) & $1.47_{-0.09}^{+0.15}$ & $1.28_{-0.11}^{+0.08}$ & $3.6_{-8}^{+14}$ & $345_{-217}^{+545}$ & $32.7_{-0.1}^{+0.1}$ \\
SEOBNR_NSBH & $1.50_{-0.11}^{+0.16}$ & $1.26_{-0.11}^{+0.10}$ & $3.4_{-9}^{+17}$ & $301_{-189}^{+518}$ & $32.7_{-0.1}^{+0.1}$ \\
\hline \hline
\end{tabular}

individual tidal parameters, and since $\Lambda_{1}$ is fixed to zero in the prior for SEOBNR_NSBH but not for the BNS models, the priors on $\tilde{\Lambda}$ for the BNS and NSBH models are quite different. In order to make a fair comparison between the posteriors on $\tilde{\Lambda}$, we divide each posterior by the prior on $\tilde{\Lambda}$, effectively obtaining a flat prior on $\tilde{\Lambda}$, as was done in Ref. [3]. We give the median and $90 \%$ credible intervals for the masses, $\chi_{\text {eff }}, \tilde{\Lambda}$, and matched filter network SNR, in Table VI.

\section{CONCLUSION}

In this work we have built an aligned-spin NSBH waveform model based on the EOB framework [49-51], the NRTidal approach [58,59] and NR simulations $[27,30,73,80,85]$ : SEOBNR_NSBH. In building the model, we have used final mass and spin fits from Ref. [79], the $\Lambda_{\mathrm{NS}}-C_{\mathrm{NS}}$ relations of Ref. [76], and fits for the disk mass from Ref. [34]. This model incorporates a suitable tapering of the frequency-domain waveform's amplitude in regions where tidal disruption occurs building on the amplitude model of Ref. [68], as evinced from physical considerations of tidal effects as the NS plunges into the $\mathrm{BH}$, and from NR simulations of those sources. Tidal corrections to the frequency-domain waveform's phase have been computed using the NRTidal framework. We have shown that SEOBNR_NSBH gives good agreement with NR simulations by comparing the waveforms in the frequency domain (Fig. 4) and time domain (Fig. 5), as well as by computing the unfaithfulnesses shown in Tables III and IV. In Fig. 6, we compare the model with two new, highly accurate, simulations from the SXS Collaboration of disruptive NSBHs with highly spinning BHs, which we used for validation. We find very good agreement across a large number of cycles. We also performed the same comparisons with the recently published model PhenomNSBH and find similar levels of agreement with NR. In Figs. 8 and 9, we have demonstrated that the model can be used to infer properties of NSBH systems using software injections and that at a large enough SNR assuming the wrong source class can lead to biased astrophysical inferences. Finally, we have reanalyzed GW170817 with the hypothesis that it is a NSBH instead of a BNS. In Table VI, we see the results are broadly consistent, although there seems to be a slight preference for smaller tidal deformability and unequal masses when recovering with SEOBNR_NSBH.

In the future, we plan to extend and improve the SEOBNR_NSBH waveform model in various ways. A relatively simple, but important, extension is to incorporate information from modes beyond the quadrupolar one using SEOBNRv4HM [109] as a baseline. This is particularly relevant, since the NS can be tidally disrupted also in cases in which the mass ratio is larger than one and the $\mathrm{BH}$ spin is large. Another crucial improvement is to extend the model to precessing NSBH binaries, since some astrophysical scenarios predict that the $\mathrm{BH}$ spin may be misaligned with respect to the orbital angular momentum. As more high-quality NR simulations of NSBHs become available, it will also be possible to develop a more accurate model for the transition from disruptive to nondisruptive mergers. It will also be interesting to study the effect of using different tidal models in order to quantify uncertainty in the tidal part of the waveform. Finally, as we have mentioned, there is currently no model that smoothly covers the full range of source classes: BBH, NSBH, and BNS. Building a model which can capture all of relevant physics is an important future goal.

\section{ACKNOWLEDGMENTS}

We would like to thank Koutarou Kyutoku and Masaru Shibata for providing us with the numerical-relativity waveforms from the SACRA code. We would like to thank Frank Ohme, Jonathan Thompson, Edward Fauchon-Jones, and Shrobana Ghosh for reviewing the LAL implementation of the SEOBNR_NSBH waveform model. We are grateful to Katerina Chatziioannou for comments on the manuscript and Luca Prudenzi for useful discussions. T. D. acknowledges support by the European Unions Horizon 2020 research and innovation program under Grant Agreement No. 749145, BNS mergers. T. H. acknowledges support from Nederlandse Organisatie voor Wetenschappelijk Onderzoek (NWO) Projectruimte grant GW-EM NS and the DeltaITP and thanks the Yukawa International Seminar (YKIS) 2019 "Black Holes and Neutron Stars with Gravitational Waves.” F. F. gratefully acknowledges support from the U.S. National Science Foundation (NSF) through Grant No. PHY-1806278. M. D. gratefully acknowledges support from the NSF through Grant No. PHY-1806207. H.P.P. gratefully 
acknowledges support from the NSERC Canada. L. E. K. acknowledges support from NSF Grants No. PHY-1606654 and No. PHY-1912081. M. A. S. acknowledge support from NSF Grants PHY170212 and PHY-1708213. L. K. and M. S. also thank the Sherman Fairchild Foundation for their support. Computations for the review were done on the Hawk high-performance compute (HPC) cluster at Cardiff University, which is funded by STFC Grant No. ST/I006285/1. Other computations for this work were done on the HPC clusters Hypatia at the Max Planck Institute for Gravitational Physics in Potsdam, and at CIT at Caltech, funded by National Science Foundation Grants No. PHY-0757058 and No. PHY-0823459. We extensively used the numpy [110], scipy [111], and matplotlib [112] libraries. This research has made use of data, software and/ or web tools obtained from the Gravitational Wave Open Science Center, a service of LIGO Laboratory, the LIGO Scientific Collaboration and the Virgo Collaboration. LIGO is funded by the U.S. National Science Foundation. Virgo is funded by the French Centre National de Recherche Scientifique (CNRS), the Italian Istituto Nazionale della Fisica Nucleare (INFN) and the Dutch Nikhef, with contributions by Polish and Hungarian institutes.

\section{APPENDIX: EXPLICIT FORM OF AMPLITUDE CORRECTION}

The amplitude corrections are parameterized based on the model presented in Ref. [68]. We use the same parametric form for each component of the amplitude correction and refit the coefficients. We have streamlined the notation.

\section{Nondisruptive}

The nondisruptive window function given in Eq. (12) contains the parameters $f_{\mathrm{ND}}, \sigma_{\mathrm{ND}}$, and $\epsilon_{\mathrm{ND}}$, which we compute as

$$
\begin{aligned}
& f_{\mathrm{ND}}=f_{\mathrm{RD}}, \\
& \sigma_{\mathrm{ND}}=\bar{\sigma}_{\mathrm{ND}}+2 w^{-}\left(x ; x_{0}, \sigma_{x}\right), \\
& \epsilon_{\mathrm{ND}}=w^{+}\left(y ; y_{0}, \sigma_{y}\right),
\end{aligned}
$$

where $f_{\mathrm{RD}}$ is the ringdown frequency, which we estimate in terms of the final mass and spin using the fits from Ref. [78]. Following Refs. [68,113], we have introduced $x$ and $y$, which are a measure of how close the merger is to becoming disruptive. These quantities appear inside of window functions in order to ensure that the corrections to the ringdown are smoothly turned off $\left(\epsilon_{\mathrm{ND}} \rightarrow 0, \sigma_{\mathrm{ND}} \rightarrow \infty\right.$ ) as the merger becomes less disruptive and therefore more like a BBH. For large $\sigma_{\mathrm{ND}}$ and $\epsilon_{\mathrm{ND}}$ on the intrinsic parameters of the binary. are determined by the tidal frequency, ringdown frequency, NS compactness, and BH spin via

$$
\begin{aligned}
& x=\left(\frac{f_{\mathrm{RD}}-f_{\text {tide }}}{f_{R D}}\right)^{2}+x_{C} C_{\mathrm{NS}}+x_{\chi} \chi_{\mathrm{BH}}, \\
& y=\left(\frac{f_{\mathrm{RD}}-f_{\text {tide }}}{f_{R D}}\right)^{2}+y_{C} C_{\mathrm{NS}}+y_{\chi} \chi_{\mathrm{BH}} .
\end{aligned}
$$

The nine coefficients $\left\{\bar{\sigma}_{\mathrm{ND}}, x_{0}, \sigma_{x}, x_{C}, x_{\chi}, y_{0}, \sigma_{y}, y_{C}, y_{\chi}\right\}$ were determined by a fitting procedure as described in Sec. II D. Their values are given in Table VII.

\section{Disruptive}

The disruptive window correction in Eq. (13) is defined in terms of $f_{\mathrm{D}}$ and $\sigma_{\mathrm{D}}$, which we parameterize as

$f_{\mathrm{D}}=\left(a_{0}+a_{M} \frac{M_{b, \text { torus }}}{M_{b, \mathrm{NS}}}+a_{C} C_{\mathrm{NS}}+a_{\nu} \sqrt{\nu}+a_{\chi} \chi\right) f_{\text {tide }}$,

$\sigma_{\mathrm{D}}=b_{0}+b_{M} \frac{M_{b, \text { torus }}}{M_{b, \mathrm{NS}}}+b_{C} C_{\mathrm{NS}}+b_{\nu} \sqrt{\nu}+\sum_{k=1}^{3} b_{\chi}^{(k)} \chi^{k}$.

In this expression, there are 12 coefficients $\left\{a_{0}, a_{M}, a_{C}\right.$, $\left.a_{\nu}, a_{\chi}, b_{0}, b_{M}, b_{C}, b_{\nu}, b_{\chi}^{(1)}, b_{\chi}^{(2)}, b_{\chi}^{(3)}\right\}$ which were determined by a fitting procedure as described in Sec. II D. Their values are given in Table VII. As in the case of Ref. [68], we find that the parameters $b_{\chi}^{(1)}, b_{\chi}^{(2)}$, and $b_{\chi}^{(3)}$ do not decrease monotonically.

TABLE VII. Parameters for the amplitude correction $w_{\text {corr }}(f)$. The parameters above the line appear in the correction for tidally disruptive mergers, and the parameters below the line appear in the correction for disruptive mergers.

\begin{tabular}{lc}
\hline \hline Parameter & Value \\
\hline $\bar{\sigma}_{\mathrm{ND}}$ & 0.0225006 \\
$x_{0}$ & -0.0923660 \\
$\sigma_{x}$ & 0.0187155 \\
$x_{C}$ & -0.486533 \\
$x_{\chi}$ & -0.0314394 \\
$y_{0}$ & -0.177393 \\
$\sigma_{y}$ & 0.771910 \\
$y_{C}$ & 0.493376 \\
$y_{\chi}$ & 0.0569155 \\
$a_{0}$ & 1.27280 \\
$a_{M}$ & -1.68735 \\
$a_{C}$ & -1.43369 \\
$a_{\nu}$ & -0.510033 \\
$a_{\chi}$ & 0.280002 \\
$b_{0}$ & 0.185326 \\
$b_{M}$ & -0.253476 \\
$b_{C}$ & 0.251061 \\
$b_{\nu}$ & -0.284595 \\
$b_{\chi}^{(1)}$ & -0.000757100 \\
$b_{\chi}^{(2)}$ & 0.018089075 \\
$b_{\chi}^{(3)}$ & 0.028545184 \\
\hline \hline
\end{tabular}


[1] J. Aasi et al. (LIGO Scientific Collaboration), Classical Quantum Gravity 32, 074001 (2015).

[2] F. Acernese et al. (Virgo Collaboration), Classical Quantum Gravity 32, 024001 (2015).

[3] B. P. Abbott et al. (LIGO Scientific and Virgo Collaborations), Phys. Rev. X 9, 031040 (2019).

[4] B. Abbott et al. (LIGO Scientific and Virgo Collaborations), arXiv:2001.01761.

[5] T. Venumadhav, B. Zackay, J. Roulet, L. Dai, and M. Zaldarriaga, Phys. Rev. D 101, 083030 (2020).

[6] B. Zackay, T. Venumadhav, L. Dai, J. Roulet, and M. Zaldarriaga, Phys. Rev. D 100, 023007 (2019).

[7] B. Zackay, L. Dai, T. Venumadhav, J. Roulet, and M. Zaldarriaga, arXiv:1910.09528.

[8] J. Abadie et al. (LIGO Scientific and Virgo Collaborations), Classical Quantum Gravity 27, 173001 (2010).

[9] B. P. Abbott et al. (KAGRA, LIGO Scientific, and Virgo Collaborations), Living Rev. Relativity 21, 3 (2018).

[10] LIGO Scientific and Virgo Collaborations, GCN 25324 (2019), https://gcn.gsfc.nasa.gov/gcn3/25324.gcn3.

[11] LIGO Scientific and Virgo Collaborations, GCN 25549 (2019), https://gcn.gsfc.nasa.gov/gen3/25549.gcn3.

[12] LIGO Scientific and Virgo Collaborations, GCN 25695 (2019), https://gcn.gsfc.nasa.gov/gcn3/25695.gcn3.

[13] LIGO Scientific and Virgo Collaborations, GCN 25814 (2019), https://gcn.gsfc.nasa.gov/gen3/25814.gcn3.

[14] LIGO Scientific and Virgo Collaborations, GCN 25876 (2019), https://gcn.gsfc.nasa.gov/gcn3/25876.gcn3.

[15] LIGO Scientific and Virgo Collaborations, GCN 26350 (2019), https://gcn.gsfc.nasa.gov/gcn3/26350.gcn3.

[16] LIGO Scientific and Virgo Collaborations, GCN 26640 (2020), https://gcn.gsfc.nasa.gov/gcn3/26640.gcn3.

[17] T. Hinderer et al., Phys. Rev. D 100, 063021 (2019).

[18] M. W. Coughlin and T. Dietrich, Phys. Rev. D 100, 043011 (2019).

[19] B. P. Abbott et al. (LIGO Scientific and Virgo Collaborations), Classical Quantum Gravity 37, 045006 (2020).

[20] M.-Z. Han, S.-P. Tang, Y.-M. Hu, Y.-J. Li, J.-L. Jiang, Z.-P. Jin, Y.-Z. Fan, and D.-M. Wei, Astrophys. J. 891, L5 (2020).

[21] K. Kyutoku, S. Fujibayashi, K. Hayashi, K. Kawaguchi, K. Kiuchi, M. Shibata, and M. Tanaka, Astrophys. J. 890, L4 (2020).

[22] M. Shibata and K. Taniguchi, Living Rev. Relativity 14, 6 (2011).

[23] É. É. Flanagan, Phys. Rev. D 58, 124030 (1998).

[24] É. É. Flanagan and T. Hinderer, Phys. Rev. D 77, 021502 (2008).

[25] J. E. Vines and É. É. Flanagan, Phys. Rev. D 88, 024046 (2013).

[26] F. Pannarale, L. Rezzolla, F. Ohme, and J. S. Read, Phys. Rev. D 84, 104017 (2011).

[27] K. Kyutoku, M. Shibata, and K. Taniguchi, Phys. Rev. D 82, 044049 (2010); 84, 049902(E) (2011).

[28] F. Foucart, L. Buchman, M. D. Duez, M. Grudich, L. E. Kidder, I. MacDonald, A. Mroue, H. P. Pfeiffer, M. A. Scheel, and B. Szilagyi, Phys. Rev. D 88, 064017 (2013).

[29] F. Foucart, M. D. Duez, L. E. Kidder, and S. A. Teukolsky, Phys. Rev. D 83, 024005 (2011).
[30] K. Kyutoku, H. Okawa, M. Shibata, and K. Taniguchi, Phys. Rev. D 84, 064018 (2011).

[31] F. Foucart, M. B. Deaton, M. D. Duez, L. E. Kidder, I. MacDonald, C. D. Ott, H. P. Pfeiffer, M. A. Scheel, B. Szilagyi, and S. A. Teukolsky, Phys. Rev. D 87, 084006 (2013).

[32] K. Kawaguchi, K. Kyutoku, H. Nakano, H. Okawa, M. Shibata, and K. Taniguchi, Phys. Rev. D 92, 024014 (2015).

[33] F. Pannarale, A. Tonita, and L. Rezzolla, Astrophys. J. 727, 95 (2011).

[34] F. Foucart, Phys. Rev. D 86, 124007 (2012).

[35] G. Lovelace, M. D. Duez, F. Foucart, L. E. Kidder, H. P. Pfeiffer, M. A. Scheel, and B. Szilágyi, Classical Quantum Gravity 30, 135004 (2013).

[36] K. Kawaguchi, K. Kyutoku, H. Nakano, and M. Shibata, arXiv:1709.02754.

[37] F. Pannarale, E. Berti, K. Kyutoku, B. D. Lackey, and M. Shibata, Phys. Rev. D 92, 081504 (2015).

[38] B. Paczynski, Astrophys. J. Lett. 308, L43 (1986).

[39] D. Eichler, M. Livio, T. Piran, and D. N. Schramm, Nature (London) 340, 126 (1989).

[40] B. Paczynski, Acta Astron. 41, 257 (1991), http://adsabs .harvard.edu/full/1991AcA....41..257P.

[41] P. Meszaros and M. Rees, Astrophys. J. 397, 570 (1992).

[42] R. Narayan, B. Paczynski, and T. Piran, Astrophys. J. Lett. 395, L83 (1992).

[43] E. Nakar, Phys. Rep. 442, 166 (2007).

[44] B. D. Metzger, Living Rev. Relativity 20, 3 (2017).

[45] M. Tanaka, Adv. Astron. 2016, 1 (2016).

[46] B. P. Abbott et al. (LIGO Scientific, Virgo, Fermi GBM, INTEGRAL, IceCube, AstroSat Cadmium Zinc Telluride Imager Team, IPN, Insight-Hxmt, ANTARES, Swift, AGILE Team, 1M2H Team, Dark Energy Camera GWEM, DES, DLT40, GRAWITA, Fermi-LAT, ATCA, ASKAP, Las Cumbres Observatory Group, OzGrav, DWF (Deeper Wider Faster Program), AST3, CAASTRO, VINROUGE, MASTER, J-GEM, GROWTH, JAGWAR, CaltechNRAO, TTU-NRAO, NuSTAR, Pan-STARRS, MAXI Team, TZAC Consortium, KU, Nordic Optical Telescope, ePESSTO, GROND, Texas Tech University, SALT Group, TOROS, BOOTES, MWA, CALET, IKIGW Follow-up, H.E.S.S., LOFAR, LWA, HAWC, Pierre Auger, ALMA, Euro VLBI Team, Pi of Sky, Chandra Team at McGill University, DFN, ATLAS Telescopes, High Time Resolution Universe Survey, RIMAS, RATIR, SKA South Africa/MeerKAT), Astrophys. J. 848, L12 (2017).

[47] T. Damour and A. Nagar, Phys. Rev. D 80, 084035 (2009).

[48] T. Binnington and E. Poisson, Phys. Rev. D 80, 084018 (2009).

[49] A. Buonanno and T. Damour, Phys. Rev. D 59, 084006 (1999).

[50] A. Buonanno and T. Damour, Phys. Rev. D 62, 064015 (2000).

[51] T. Damour, P. Jaranowski, and G. Schfer, Phys. Rev. D 62 , 084011 (2000).

[52] T. Damour and A. Nagar, Phys. Rev. D 81, 084016 (2010). 
[53] T. Damour, A. Nagar, and L. Villain, Phys. Rev. D 85, 123007 (2012).

[54] J. Steinhoff, T. Hinderer, A. Buonanno, and A. Taracchini, Phys. Rev. D 94, 104028 (2016).

[55] T. Hinderer et al., Phys. Rev. Lett. 116, 181101 (2016).

[56] A. Nagar et al., Phys. Rev. D 98, 104052 (2018).

[57] B. D. Lackey, M. Pürrer, A. Taracchini, and S. Marsat, Phys. Rev. D 100, 024002 (2019).

[58] T. Dietrich, S. Bernuzzi, and W. Tichy, Phys. Rev. D 96, 121501 (2017).

[59] T. Dietrich, A. Samajdar, S. Khan, N. K. JohnsonMcDaniel, R. Dudi, and W. Tichy, Phys. Rev. D 100, 044003 (2019).

[60] A. Bohé et al., Phys. Rev. D 95, 044028 (2017).

[61] S. Khan, S. Husa, M. Hannam, F. Ohme, M. Pürrer, X. Jimenez Forteza, and A. Bohé, Phys. Rev. D 93, 044007 (2016).

[62] M. Hannam, P. Schmidt, A. Bohé, L. Haegel, S. Husa, F. Ohme, G. Pratten, and M. Pürrer, Phys. Rev. Lett. 113, 151101 (2014).

[63] B. D. Lackey, K. Kyutoku, M. Shibata, P. R. Brady, and J. L. Friedman, Phys. Rev. D 85, 044061 (2012).

[64] B. D. Lackey, K. Kyutoku, M. Shibata, P. R. Brady, and J. L. Friedman, Phys. Rev. D 89, 043009 (2014).

[65] P. Kumar, M. Pürrer, and H. P. Pfeiffer, Phys. Rev. D 95, 044039 (2017).

[66] A. Taracchini et al., Phys. Rev. D 89, 061502 (2014).

[67] J. E. Thompson, E. Fauchon-Jones, S. Khan, E. Nitoglia, F. Pannarale, T. Dietrich, and M. Hannam, Phys. Rev. D 101, 124059 (2020).

[68] F. Pannarale, E. Berti, K. Kyutoku, B. D. Lackey, and M. Shibata, Phys. Rev. D 92, 084050 (2015).

[69] LIGO Scientific Collaboration, LALSuite: LSC Algorithm Library Suite, https://www.lsc-group.phys.uwm (2020).

[70] L. Santamaria et al., Phys. Rev. D 82, 064016 (2010).

[71] M. Pürrer, Classical Quantum Gravity 31, 195010 (2014).

[72] M. Pürrer, Phys. Rev. D 93, 064041 (2016).

[73] F. Foucart et al., Phys. Rev. D 99, 044008 (2019).

[74] L. G. Fishbone, Astrophys. J. 185, 43 (1973).

[75] T. Binnington and E. Poisson, Phys. Rev. D 80, 084018 (2009).

[76] K. Yagi and N. Yunes, Phys. Rep. 681, 1 (2017).

[77] F. Foucart, M. D. Duez, L. E. Kidder, S. Nissanke, H. P. Pfeiffer, and M. A. Scheel, Phys. Rev. D 99, 103025 (2019).

[78] E. Berti, V. Cardoso, and C. M. Will, Phys. Rev. D 73, 064030 (2006).

[79] F. Zappa, S. Bernuzzi, F. Pannarale, M. Mapelli, and N. Giacobbo, Phys. Rev. Lett. 123, 041102 (2019).

[80] F. Foucart, L. Buchman, M. D. Duez, M. Grudich, L. E. Kidder, I. MacDonald, A. Mroue, H. P. Pfeiffer, M. A. Scheel, and B. Szilagyi, Phys. Rev. D 88, 064017 (2013).

[81] Spectral Einstein Code, https://www.black-holes.org/code/ SpEC.html (2018).

[82] M. D. Duez, F. Foucart, L. E. Kidder, H. P. Pfeiffer, M. A. Scheel, and S. A. Teukolsky, Phys. Rev. D 78, 104015 (2008).

[83] F. Foucart, M. B. Deaton, M. D. Duez, L. E. Kidder, I. MacDonald, C. D. Ott, H. P. Pfeiffer, M. A. Scheel,
B. Szilagyi, and S. A. Teukolsky, Phys. Rev. D 87, 084006 (2013).

[84] J. S. Read, B. D. Lackey, B. J. Owen, and J. L. Friedman, Phys. Rev. D 79, 124032 (2009).

[85] F. Foucart, M. B. Deaton, M. D. Duez, E. O'Connor, C. D. Ott, R. Haas, L. E. Kidder, H. P. Pfeiffer, M. A. Scheel, and B. Szilagyi, Phys. Rev. D 90, 024026 (2014).

[86] J. M. Lattimer and F. D. Swesty, Nucl. Phys. A535, 331 (1991).

[87] T. Yamamoto, M. Shibata, and K. Taniguchi, Phys. Rev. D 78, 064054 (2008).

[88] T. Dietrich et al., Phys. Rev. D 99, 024029 (2019).

[89] J. A. Nelder and R. Mead, Comput. J. 7, 308 (1965).

[90] T. Damour, B. R. Iyer, and B. Sathyaprakash, Phys. Rev. D 57, 885 (1998).

[91] B. S. Sathyaprakash and S. V. Dhurandhar, Phys. Rev. D 44, 3819 (1991).

[92] L. S. Finn and D. F. Chernoff, Phys. Rev. D 47, 2198 (1993).

[93] L. Barsotti, P. Fritschel, M. Evans, and S. Gras, Updated Advanced LIGO sensitivity design curve, https://dcc.ligo .org/LIGO-T1800044/public (2018).

[94] M. Boyle et al., Classical Quantum Gravity 36, 195006 (2019).

[95] B. Abbott et al. (LIGO Scientific and Virgo Collaborations), Phys. Rev. Lett. 121, 161101 (2018).

[96] K. Arun, A. Buonanno, G. Faye, and E. Ochsner, Phys. Rev. D 79, 104023 (2009); 84, 049901(E) (2011)].

[97] K. K. Ng, S. Vitale, A. Zimmerman, K. Chatziioannou, D. Gerosa, and C.-J. Haster, Phys. Rev. D 98, 083007 (2018).

[98] M. Hannam, D. A. Brown, S. Fairhurst, C. L. Fryer, and I. W. Harry, Astrophys. J. Lett. 766, L14 (2013).

[99] L. Lindblom, B. J. Owen, and D. A. Brown, Phys. Rev. D 78, 124020 (2008).

[100] K. Chatziioannou, A. Klein, N. Yunes, and N. Cornish, Phys. Rev. D 95, 104004 (2017).

[101] M. Pürrer and C.-J. Haster, Phys. Rev. Research 2, 023151 (2020).

[102] S. Nissanke, D. E. Holz, S. A. Hughes, N. Dalal, and J. L. Sievers, Astrophys. J. 725, 496 (2010).

[103] J. Veitch et al., Phys. Rev. D 91, 042003 (2015).

[104] Y. Huang, C.-J. Haster, S. Vitale, V. Varma, F. Foucart, and S. Biscoveanu, arXiv:2005.11850.

[105] K. Barkett, Y. Chen, M. A. Scheel, and V. Varma, Phys. Rev. D 102, 024031 (2020).

[106] B. P. Abbott et al. (LIGO Scientific and Virgo Collaborations), Phys. Rev. Lett. 119, 161101 (2017).

[107] M. Vallisneri, J. Kanner, R. Williams, A. Weinstein, and B. Stephens, J. Phys. Conf. Ser. 610, 012021 (2015).

[108] R. Abbott et al. (LIGO Scientific and Virgo Collaborations), arXiv:1912.11716.

[109] R. Cotesta, A. Buonanno, A. Bohé, A. Taracchini, I. Hinder, and S. Ossokine, Phys. Rev. D 98, 084028 (2018).

[110] T. E. Oliphant, A Guide to NumPy (Trelgol Publishing, USA, 2006).

[111] P. Virtanen, R. Gommers, T. E. Oliphant, M. Haberland, T. Reddy, D. Cournapeau, E. Burovski, P. Peterson, W. Weckesser, J. Bright et al., Nat. Methods 17, 261 (2020).

[112] J. D. Hunter, Comput. Sci. Eng. 9, 90 (2007).

[113] F. Pannarale, E. Berti, K. Kyutoku, and M. Shibata, Phys. Rev. D 88, 084011 (2013). 